\title{
A stochastic model for chain collisions of vehicles equipped with vehicular communications
}

\author{
Carolina Garcia-Costa, Esteban Egea-Lopez, Juan Bautista Tomas-Gabarron, Joan Garcia- \\ Haro, Member, IEEE, and Zygmunt J. Haas, Fellow, IEEE
}

\begin{abstract}
Improvement of traffic safety by cooperative vehicular applications is one of the most promising benefits of VANET. However, in order to develop properly such applications, the influence of the different driving parameters on the event of a vehicle collision must be assessed at an early design stage. In this paper we derive a stochastic model for the number of accidents in a platoon of vehicles equipped with a warning collision notification system, which is able to inform all the vehicles about an emergency event. In fact, the assumption of communications being used is key to simplify the derivation of a stochastic model. The model enables the computation of the average number of collisions that occur in the platoon, the probabilities of the different ways in which the collisions may take place as well as other statistics of interest. Although an exponential distribution has been used for the traffic density, it is also valid for different probability distributions for the traffic densities as well as for other significant parameters of the model. Moreover, the actual communication system employed is independent of the model since it is abstracted by a message delay variable, which allows it to be used to evaluate different communication technologies. We validate the proposed model with Monte-Carlo simulations. With this model one can quickly evaluate numerically the influence of the different model parameters (vehicle density, velocities, decelerations and delays) on the collision process and draw conclusions that shed relevant guidelines for the design of vehicular communication systems as well as Chain Collision Avoidance (CCA) applications. Illustrative examples of application are provided, though a systematic characterization and evaluation of different scenarios is left as future work.
\end{abstract}

Index Terms - Vehicle safety, vehicular communications, chain collision, vehicle platoon, collision avoidance, stochastic model, road accidents

\section{INTRODUCTION}

$\mathbf{I}$ NTER-VEHICLE communications based on wireless technologies pave the way for novel applications in traffic

Manuscript received March 23, 2011; revised XX XX, 2011. This research has been supported by the MICINN/FEDER project grant TEC201021405-C02-02/TCM (CALM) and Fundación Seneca RM grant 00002/CS/08 FORMA. It is also developed in the framework of "Programa de Ayudas a Grupos de Excelencia de la Región de Murcia, de la Fundación Séneca, Agencia de Ciencia y Tecnología de la RM". J. Garcia-Haro acknowledges personal grant PR2009-0337. J. B. Tomas-Gabarron thanks the Spanish MICINN for a FPU (REF AP2008-02244) pre-doctoral fellowship. C. GarciaCosta also acknowledges the Fundación Seneca for a FPI (REF 12347/FPI/09) pre-doctoral fellowship. E. Egea acknowledges UPCT for a PMPDI-UPCT2011 grant.

C. Garcia-Costa, E. Egea-Lopez, J. B. Tomas-Gabarron and J. GarciaHaro are with the Department of Information and Communication Technologies, Universidad Politécnica de Cartagena (UPCT), Spain, e-mail: esteban.egea@upct.es.

Z. J. Haas is with the School of Electrical and Computer Engineering, Wireless Networks Lab. Cornell University, Ithaca, NY, USA. safety, driver-assistance, traffic control and other advanced services which will make future Intelligent Transportation Systems (ITS). The advances in technology and standardization, especially with the allocation of dedicated bandwidth to vehicular communications, from the mid 1990s have increased research and development efforts on Vehicular AdHoc Networks (VANET) from the networking and mobile communications community [1], though early research on "Automated Highways Systems" goes back to the 1960s and later [2]. Improvement of traffic safety by cooperative vehicular applications is one of the most promising technical and social benefits of VANET [3], [4]. However, in order to design and implement such applications, a deep understanding of the vehicle collision processes is needed. The influence of the different driving parameters on the collision event must be assessed at an early design stage in order to develop applications that can timely adapt vehicle dynamics to avoid or at least mitigate the danger [5].

Very detailed models of vehicle motion and collision dynamics can be found [6], [7], but the equations are completely deterministic, whereas, in reality, randomness is always present as an effect of human behaviour or noisy operation introduced by sensors or other reasons. To account for it, the usual methodology is to evaluate deterministic models by applying a Monte-Carlo or stochastic analysis over an extensive range of their parameters [2], [6], [8]. However, to the authors' knowledge, little effort has been devoted to develop models which are stochastic in nature, and in particular for rear-end chain collisions of vehicles. Some reasons behind it are the difficulties of evaluating all the possible ways in which a collision may occur and the complexity posed by the fact that the motion equations for those possibilities involve a dependence on the parameters of preceding vehicles. That is, the driver reacts to variations in the driving conditions of the preceding vehicle, as in a car-following approach [9], [10]. However, if vehicles use a communication system which is able to inform all the vehicles about an emergency event, those difficulties can be overcome. The key is that, in that case, it can be assumed that drivers react as soon as they receive a warning message and they start braking independently of the preceding vehicles behavior. This is in fact the goal of warning collision systems or Electronic Brake Warning (EBW) applications. This assumption removes the dependence of the motion equations on the preceding vehicles and facilitates the development of a stochastic model.

In this paper we take this approach. Our goal is to describe 
and analyze the risk of colliding for a set of moving vehicles forming a platoon (or chain) and equipped with a warning collision system when there is a sudden stop of the leading vehicle. To this aim, we derive a stochastic model for the number of accidents that occurs in this scenario. The model allows us to compute the average number of collisions that occur in the platoon, among other statistics of interest. The scenario under consideration is basically a platoon of vehicles moving along a unidimensional road in the same direction in which the leading vehicle suddenly comes to a complete stop. To consider a worst case scenario we add two strong assumptions: first, the leading vehicle stops instantly (it may also be considered that a fixed obstacle lays on the road). Second, vehicles will not be able to change their direction of movement to cope with the unexpected incident. Our model is stochastic because all its parameters may be described by random variables. We derive the equations assuming always a random intervehicle spacing, in particular for an exponentially distributed spacing, though the model is valid for other distributions. When additional parameters are assumed random, the solutions have been computed numerically. Additionally, it should be observed that the model is independent of the communication technology, since the operation of the communication system is abstracted by the use of a message reception/notification delay variable. Finally, the probabilities for all the ways the collision may take place are also derived, which can be further used to evaluate the severity of accidents in higher detail, for instance, by assigning different severity weights to different types of collision. A deeper discussion on this topic, however, is out of the scope of this paper.

The main practical utility of this model lays in its ability to quickly evaluate numerically the influence of the different parameters on the collision process, without the need to resort to complex simulations in a first stage. Such an evaluation provides relevant guidelines for the design of vehicular communication systems as well as Chain Collision Avoidance (CCA) applications. As an example, it can quickly reveal for which range and distributions of the parameters the communication delay has a serious impact on the metric of interest, which can be the average number of accidents but also the probability of collision of every vehicle in the chain. Since it turns out that in some scenarios a low delay is not relevant for the outcome, a communication system could trade it off for additional reliability mechanisms. Moreover, in this paper we set either constant or purely random parameters, but the model can be used with arbitrary parameters to evaluate more specific applications. For instance, to evaluate multihop communications we can set up a vector of delays with progressively increasing values. We provide examples of use in Section V, but in any case, a careful characterization of the model parameters for the scenarios and applications is a necessary previous step.

So, in summary, in this paper our goal is to provide the derivation and validation of the model and show its utility with a few illustrative examples. A proper characterization and evaluation of different scenarios and metrics is left as future work.

The remainder of this paper is organized as follows. In
Section II we briefly review the related work. The derivation and validation of the model is provided in Sections III and IV. Section V illustrates how to use the model to evaluate the influence of different parameters on the collision process and to obtain qualitative conclusions relevant to the design of vehicular communication as well as CCA applications. Conclusions and future work are remarked in Section VI, while the necessary auxiliary material is relegated to the Appendices $\mathrm{A}$ and $\mathrm{B}$.

\section{RELATED WORK}

Our model assumes that there is a communication system between vehicles that allows them to receive warning messages to start braking in the event of a sudden stop of the leading car. However, such a system is abstracted in the model and characterized by the use of a message reception/notification delay variable. Therefore, our model is actually independent of it and can be applied to any communication system whose operation can be abstracted by an appropriate delay variable. For instance, current VANET standards specify the use of IEEE 802.11p which is based on contention (CSMA) Medium Access Control [1]. Such a shared channel MAC technique can be abstracted in our model by a delay random variable with an appropriate probability distribution [11]. Further details on current VANET communication technologies can be found in [1].

Regarding collision models for chains of vehicles two different groups of studies can be found: (i) statistical models of the frequency of accidents occurrence and their circumstances [12], [13], and (ii) models of the collision process itself based on physical parameters [2], [6], [8]. This paper falls on the latter category and additionally assumes that an automated warning system is in place. In most of these studies deterministic equations for the occurrence of collisions are derived and, to account for random variability, stochastic analysis or MonteCarlo simulations over a wide range of model parameters are performed afterwards to obtain an estimate of the collision probability or other metrics of interest. Our approach is different and the model shown here is directly stochastic and assumes that at least the inter-vehicle distance is a random variable, which is in fact a realistic assumption as shown in [14]. We also perform Monte-Carlo simulations but, unlike the previously mentioned papers, we use them to validate our model rather than to obtain metrics of interest. Looking into these works in particular, in an early study Fenton [2] defines an accident cost function to evaluate the severity of vehicle collisions. The collision model used is derived for an automatically controlled ${ }^{1}$ platoon of vehicles which advance at constant speed with a constant inter-vehicle spacing. A more recent work [8] provides a similar collision model for a four-car platoon of vehicles assuming that just one of the vehicles is equipped with an autonomous intelligent cruise control. In both cases, the collision model defines how vehicles decelerate in order to obtain a deterministic equation for the

\footnotetext{
${ }^{1}$ Let us note that early research, which goes back to the 1960 s, considered the hypothesis of achieving "automated highway systems", where most of the driving tasks were automatically controlled.
} 
collision. Afterwards, the evaluation is done by randomizing some parameters of the model and running a Monte-Carlo simulation. In [10], authors derive conditions necessary for a chain collision, starting from a car-following model. However, they assume that all the vehicles are driving with equal initial speeds and inter-vehicle distances.

Interestingly, the vehicle collision model proposed in this paper is more general, it explicitly accounts for random intervehicle spacing, and can be used to assign arbitrary variables, even random ones, to the kinematic parameters of each vehicle as well as the warning message communication delay. Moreover, there are additional applications of our model, for instance, it can be readily used to evaluate the severity of collisions, as in [2]: since we compute the probability of collisions occurring in several manners, we could assign a severity weight to each possibility, that is, we may assign more severity to a collision when both vehicles are in motion than to other cases, for example, though this topic is not treated in the present paper. On the other hand, some of the results in [2] are similar to ours, for instance the sensitivity shown to the decrease in deceleration capabilities of the subsequent vehicles. In all the cases as well as in this paper, only rear-end collisions are considered. Head-on collisions are evaluated in [6], based on a very detailed analytical model of the vehicle.

Finally, in this paper we provide examples about the kind of results that can be drawn from the proposed model which are useful for the design of CCA applications. A review on intelligent collision avoidance algorithms can be found in [5]. In particular, the influence of delay notification on different scenarios is useful to set appropriate time horizons for CCA systems based on trajectory prediction [3].

\section{COLLISION MODEL}

We consider a platoon (or chain) of $N$ vehicles following a leading one (see Fig. 1), where each vehicle $C_{i}, i \in 1 \ldots N$, moves at constant velocity $V_{i}$. The leading vehicle $C_{0}$ collides with an obstacle on the road, at time $t_{0}=0$, and immediately it sends a warning message to the following vehicles. The rest of the vehicles start to brake at constant deceleration ${ }^{2}$ $a_{i}$ when they are aware of the risk of collision, that is, after a time lapse $\delta_{i}$. Let us remark here that this time lapse is mainly determined by the reception of the warning message, generated by the communication system, so the reaction of the driver is independent of the movement state of the preceding vehicle. That is, a warned driver will decelerate even if the preceding car has not started to decelerate. In a classical carfollowing approach, on the contrary, the deceleration would be a consequence of a change in the speed or inter-vehicle spacing of the preceding vehicle. For the sake of simplicity, we assume that every vehicle has the same length $L$ and its position is given by the $x$ coordinate of its front bumper. The leading vehicle stops at coordinate $x_{0}=0$ and the initial intervehicle spacing is $s_{i}=x_{i}-\left(x_{i-1}+L\right)$. We assume that at least the inter-vehicle spacing is a random variable. Its probability distribution as well as the variability of the other variables $a_{i}$,

\footnotetext{
${ }^{2}$ To simplify the notation, in the remaining of the paper we consider $a_{i}$ a deceleration, and so assign it a positive sign.
}

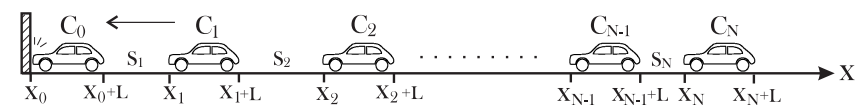

Fig. 1. The scenario under consideration.
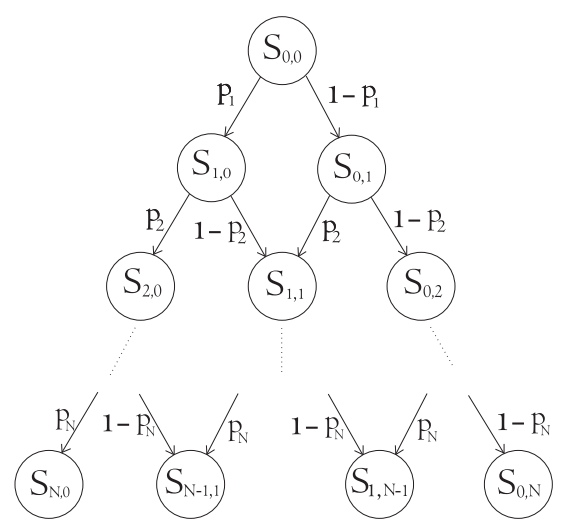

Fig. 2. Probability tree diagram that defines the model. $S_{i, j}$ represents the state with $i$ collided vehicles and $j$ successfully stopped vehicles.

$V_{i}, \delta_{i}$ is discussed in Sect. IV. Vehicles cannot change lane or perform evasive maneuvers.

With this model the final outcome of a vehicle depends on the outcome of the preceding vehicles. Therefore, the collision model is based on the construction of the probability tree depicted in Fig. 2. We consider an initial state in which no vehicle has collided. Once the danger of collision has been detected, the first vehicle in the chain $C_{1}$ (immediately after the leading one) may collide or stop successfully. From both of these states two possible cases spring as well, that is either the following vehicle in the chain $C_{2}$ may collide or stop successfully. And so on until the last vehicle in the chain denoted by $C_{N}$. At the last level of the probability tree there are $N+1$ possible outcomes (final outcomes) which represent the number of collided vehicles, that is, from 0 to $N$ possible collisions. Observe that $S_{i, j}$ represents the state with $i$ collided vehicles and $j$ successfully stopped vehicles.

The transition probability between the nodes of the tree is the probability of collision of the corresponding vehicle in the chain $p_{i}$ (or its complementary). These probabilities are crucial to the model and will be calculated recursively, as described in the next section. Let us note how every path in the tree from the root to the leaves leads to a possible outcome involving every vehicle in the chain. The probability of a particular path results from the product of the transition probabilities that belong to the path. Since there are multiple paths that may lead to the same final outcome (a particular leaf node in the tree), the probability of that outcome will be the sum of the resulting probabilities of every possible path reaching it.

In order to compute the probabilities of the final outcomes, we can construct a Markov chain whose state diagram is based on the previously discussed probability tree. It is a homogeneous Markov chain with $\frac{(N+1)(N+2)}{2}$ states, $\left(S_{0,0}, S_{1,0}, S_{0,1}, \ldots, S_{N, 0}, S_{N-1,1}, \ldots, S_{1, N-1}, S_{0, N}\right)$. The transition matrix $\mathbf{P}$ of the resulting Markov chain is a square matrix of dimension $\frac{(N+1)(N+2)}{2}$, which is a sparse 


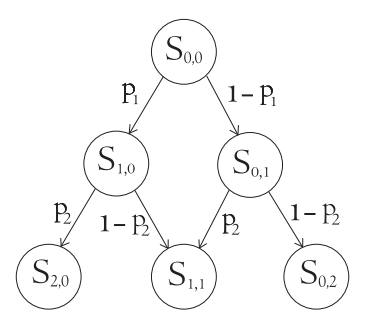

$$
P=\left(\begin{array}{cccccc}
0 & \mathrm{p}_{1} & 1-\mathrm{P}_{1} & 0 & 0 & 0 \\
0 & 0 & 0 & \mathrm{p}_{2} & 1-\mathrm{P}_{2} & 0 \\
0 & 0 & 0 & 0 & \mathrm{p}_{2} & 1-\mathrm{P}_{2} \\
0 & 0 & 0 & 0 & 0 & 0 \\
0 & 0 & 0 & 0 & 0 & 0 \\
0 & 0 & 0 & 0 & 0 & 0
\end{array}\right)
$$

Fig. 3. Probability tree and transition matrix for a chain with $N=2$ vehicles.

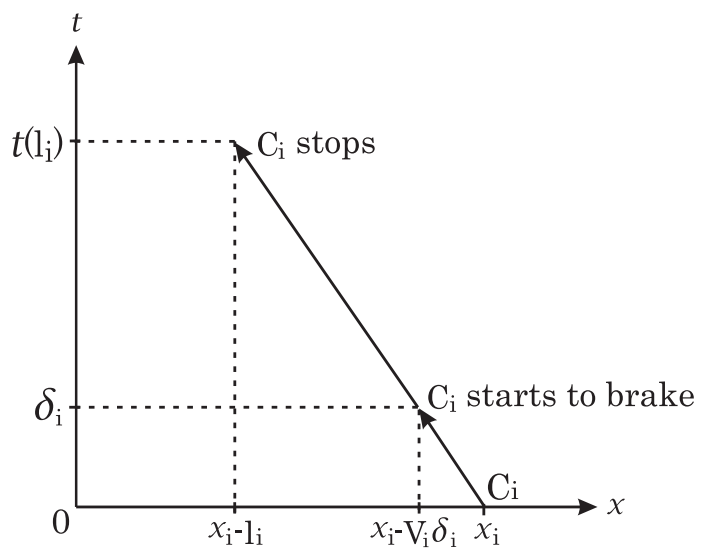

Fig. 4. Parameters of the kinematic model used to compute the vehicle collision probabilities.

matrix, since from each state it is only possible to move to two of the other states. For the sake of clarity, a brief example with 2 vehicles is illustrated in Fig. 3.

Then, we need to compute the probabilities of going from the initial state to each of the $N+1$ final states in $N$ steps, which are given by $\mathbf{P}^{N}$. Therefore, the final outcome probabilities are the last $N+1$ entries of the first row of the matrix $\mathbf{P}^{N}$.

Let $\Pi_{i}$ be the probability of reaching the final outcome with $i$ collided vehicles, that is, state $S_{i, N-i}$. Then, $\Pi_{i}=$ $\mathbf{P}^{N}\left(1, \frac{(N+1)(N+2)}{2}-i\right)$. We obtain the average of the total number of accidents in the chain using the weighted sum:

$$
N_{a c c}=\sum_{i=0}^{N} i \cdot \Pi_{i} .
$$

\section{Computation of THE Vehicle COLlision PROBABILITIES}

Computing the collision probabilities is the main problem in our model. In this section we start from a deterministic kinematic model and compute the collision probabilities when different parameters of the kinematic model are considered variables. The results are validated by Monte-Carlo simulations. Hence, we start from a basic kinematic collision model provided by [15], that can be summarized as follows.

Let $l_{i}$ represent the total distance traveled by vehicle $C_{i}$ since the emergency event occurs at time instant $t_{0}=0$ until the vehicle completely stops or collides with $C_{i-1}$. Let $\delta_{i}$ be the time lapse that goes between the detection of the emergency event until vehicle $C_{i}$ actually begins to slow down. We call $\delta_{i}$ the notification delay which models the delay between the time instant $t_{0}=0$ and the instant the driver of vehicle $C_{i}$ is aware of it and starts to brake. These parameters are depicted in Fig. 4. The notification delay plays an important role if we consider a communication system in operation between the vehicles. In this case, we can assume that the driver starts to brake when it receives a warning message, so if the emergency event occurs at $t_{0}=0$ the warning message is received at $t=\delta_{i}$ by the vehicle $C_{i}$. However, we assume a more realistic case in which there is also a reaction time before the driver actually starts to brake. Therefore $\delta_{i}=T_{m, i}+T_{r, i}$, where $T_{m, i}$ is the message reception delay and $T_{r, i}$ is the driver reaction time.

Considering a constant deceleration $a_{i}$, the distance needed by vehicle $C_{i}$ to completely stop if it does not collide is given by:

$$
d_{s, i}=\frac{V_{i}^{2}}{2 a_{i}}+V_{i} \delta_{i} .
$$

However, when a collision occurs, the actual distance traveled by the car, $d_{c, i}$, is not given by (2) anymore, but one has to consider the way the collision has occurred. For example, if a vehicle crashes, its actual distance to stop is obviously shorter than $d_{s, i}$, as illustrated in Fig. 5, and also different when both vehicles are still in motion when the crash occurs.

Let us remark at this point that (2) implies that a communication system is in place and all vehicles start to brake when they receive the message, independently of the behavior of the preceding vehicles. Otherwise, drivers would start to brake only when they sensed the braking of its nearest forward neighbor as in a car-following approach [9], [10], so (2) would become a function of the parameters of the preceding vehicle, that is, $d_{s, i}=f\left(V_{i}, V_{i-1}, a_{i}, a_{i-1}, \delta_{i}, \delta_{i-1}\right)$ and the problem would become more complex.

In all the cases the probability of collision of vehicle $C_{i}$ depends on the relationship between its distance to stop, $d_{s, i}$, the total distance traveled by the preceding vehicle, $l_{i-1}$, and the initial inter-vehicle space, $s_{i}$. That is, when $d_{s, i}<l_{i-1}+s_{i}$ the vehicle is able to stop without colliding.

At this point we also assume another simplification: if two vehicles collide we consider that they instantly stop at the point of collision. This way we keep on assuming a worst case evaluation. There are more realistic approaches, for instance, to take into account the conservation of the linear moments to compute the displacement due to the crash [7].

As can be seen from the previous equation, the number of collisions depends on the vector of velocities $V_{i}$, decelerations $a_{i}$, notification delays $\delta_{i}$, and inter-vehicle distances $s_{i}$, which we refer to as kinematic parameters. When all the parameters are given, the model is completely deterministic. However, we are interested in a more realistic case involving random variability of the parameters. To study the influence of the different parameters on collisions we introduce variability on different model parameters as follows: for all the cases we consider that $s_{i}$ is an exponentially distributed random variable with parameter $\lambda$. This parameter represents the density of vehicles on the road, defined as the average number of vehicles per meter. Let us remark that $s_{i}$ can adopt a different 


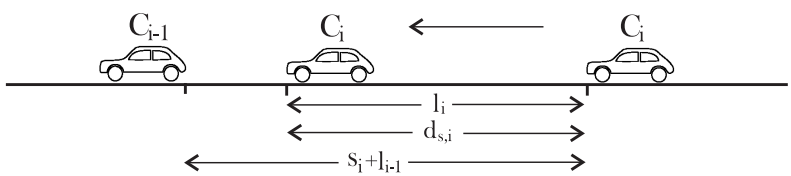

(a) Vehicle $C_{i}$ is able to stop successfully, then $l_{i}=d_{s, i}$.

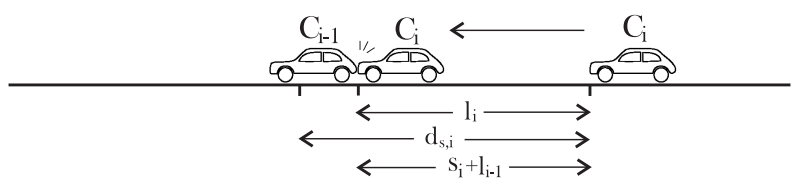

(b) Vehicle $C_{i}$ collides with $C_{i-1}$. In this case, the actual distance covered by $C_{i}$ up to the collision is shorter than $d_{s, i}$ as given by (2).

Now it is $l_{i}=s_{i}+l_{i-1}$ and depends on the distance covered by $C_{i-1}$.

Fig. 5. The distance $l_{i}$ traveled by a vehicle when there is a collision (b) is shorter than the distance needed by it to stop successfully (a), $d_{s, i}$.

distribution and the following model is still valid. The reason for this is that since $s_{i}$ is the inter-vehicle spacing when the emergency event occurs, we can consider it independent of the rest of parameters of the model, which means that the following equations would be essentially the same, but substituting the exponential probability density function by the corresponding new one. We have selected an exponential distribution because it simplifies the computations and it has been shown that describes well inter-vehicle spacing when traffic densities are small [14], whereas high traffic densities show log-normal distributions [14].

Once we have described our collision model, we next derive a basic model for the vehicle collision probabilities in which all the parameters are constant except for the inter-vehicle distance. Then, we extend the model by considering variable the rest of the kinematic parameters. This way we can evaluate the effects of the different parameters on the vehicle collision model.

\section{A. Basic model}

Our first step is to evaluate the basic model, considering all the parameters constant, except for $s_{i}$, which is assumed exponentially distributed. If a vehicle is able to stop without colliding and the kinematic parameters are constant it always travels the same distance $d_{s}$. But if there is a collision, a vehicle only travels the initial inter-vehicle distance plus the distance traveled by the preceding vehicle until it collides. Therefore, we have to compute the collision probability conditioned on the distance traveled by the previous vehicle. In the following subsections we first compute this probability exactly and then we provide an approximation that allows us to simplify the computations when additional variable parameters are considered in the model.

1) Case 1. Exact computation of collision probabilities with constant kinematic parameters: In this case we compute the collision probability exactly. For the sake of clarity, our assumptions are summarized as follows:

1) All vehicles move at the same constant velocity $V$.

2) All vehicles begin to slow down at the same constant deceleration $a$.
3) The delay $\delta$ is the same for all drivers. It implies that all the drivers receive the warning message at the same instant.

Since $V_{i}, \delta_{i}$ and $a_{i}$ are constants, from (2) we obtain:

$$
d_{s}=\frac{V^{2}}{2 a}+V \delta
$$

For $1 \leq i \leq N$, the collision probability will be computed as follows:

$$
\begin{aligned}
p_{i}= & P\left(d_{s} \geq l_{i-1}+s_{i}\right)= \\
= & P\left(l_{i-1}+s_{i} \leq d_{s} \mid l_{i-1} \leq d_{s}\right) P\left(l_{i-1} \leq d_{s}\right)+ \\
& +P\left(l_{i-1}+s_{i} \leq d_{s} \mid l_{i-1}>d_{s}\right) P\left(l_{i-1}>d_{s}\right),
\end{aligned}
$$

where $l_{i-1}$ is a random variable that represents the distance traveled by the preceding vehicle (assuming that $l_{0}=0$, since vehicle $C_{0}$ stops instantly at $x_{0}=0$ ), and $F$ is the cumulative distribution function of the exponential distribution, $\exp (\lambda)$, with $\lambda$ the vehicle density (in veh $/ m$ ).

In this simple case, if vehicle $C_{i-1}$ does not collide then neither does vehicle $C_{i}$, because the velocity, the deceleration and the reaction time are the same for both of them. Moreover, if vehicle $C_{i-1}$ collides, it means that all of the preceding vehicles have collided. From these observations we can conclude that $l_{i-1}=s_{1}+s_{2}+\ldots+s_{i-1} \sim \operatorname{Erlang}(i-1, \lambda)$, and $P\left(l_{i-1}+s_{i} \leq d_{s} \mid l_{i-1}>d_{s}\right)=0$.

Now, we need to compute $p_{i}=P\left(l_{i-1}+s_{i} \leq d_{s} \mid l_{i-1} \leq\right.$ $\left.d_{s}\right) P\left(l_{i-1} \leq d_{s}\right)$.

The joint probability density function of $X=l_{i-1}+s_{i}$ and $Y=l_{i-1}$ is:

$$
g(x, y)=\frac{\lambda^{2}(\lambda y)^{i-2} e^{-\lambda x}}{(i-2) !} \text { for } \quad 0 \leq y \leq x
$$

So, the joint cumulative distribution function is:

$$
\begin{aligned}
G(x, y) & =\int_{0}^{y} \int_{0}^{t} \frac{\lambda^{2}(\lambda s)^{i-2} e^{-\lambda t}}{(i-2) !} d s d t+ \\
& +\int_{y}^{x} \int_{0}^{y} \frac{\lambda^{2}(\lambda s)^{i-2} e^{-\lambda t}}{(i-2) !} d s d t= \\
= & \frac{\gamma(i, \lambda y)}{(i-1) !}+\frac{(\lambda y)^{i-1}}{(i-1) !}\left(e^{-\lambda y}-e^{-\lambda x}\right), \text { for } 0 \leq y \leq x .
\end{aligned}
$$

where $\gamma$ is the incomplete gamma function, defined as $\gamma(a, x)=\int_{0}^{x} t^{a-1} e^{-t} d t$.

Finally, for $1<i \leq N$ it holds:

$$
\begin{aligned}
& p_{i}=P\left(l_{i-1}+s_{i} \leq d_{s} \mid l_{i-1} \leq d_{s}\right) P\left(l_{i-1} \leq d_{s}\right)= \\
& =\frac{G\left(d_{s}, d_{s}\right)}{F_{y}\left(d_{s}\right)} \cdot F_{y}\left(d_{s}\right)=G\left(d_{s}, d_{s}\right)= \\
& =\frac{\gamma\left(i, \lambda d_{s}\right)}{(i-1) !}+\frac{\left(\lambda d_{s}\right)^{i-1}}{(i-1) !}\left(e^{-\lambda d_{s}}-e^{-\lambda d_{s}}\right)=\frac{\gamma\left(i, \lambda d_{s}\right)}{(i-1) !} .
\end{aligned}
$$

At this point, if the metric of interest is the average number of accidents, the procedure to obtain it is: once we have computed the collision probability for each vehicle, we have to construct the matrix $\mathbf{P}$ described on Section III. The next step is to calculate the final outcome probabilities, $\Pi_{i}$, and finally the average number of accidents can be obtained through (1). 
As can be seen, in this case it is relatively easy to compute the collision probability conditioned on the distance traveled by the preceding vehicle, $l_{i-1}$. However, in the following cases it becomes increasingly difficult. Besides, it can be seen that the collision probability basically depends on the difference $d_{s, i}-l_{i-1}$ of any two cars being greater than the initial intervehicle distance $s_{i}$. From this observation, and in order to simplify the following computations, in the next section we compute the collision probability using the average distance traveled by the preceding vehicle and compare it with the results of this subsection.

2) Case 2. Approximate computation of collision probabilities with constant kinematic parameters: As discussed previously, in this subsection we compute an approximation to the collision probability for the basic model, where we use the average distance traveled by the preceding vehicle, and compare it with the exact computation of Case 1. For the sake of clarity, our assumptions are summarized as follows:

1) All vehicles move at the same constant velocity $V$.

2) All vehicles begin to slow down at the same constant deceleration $a$ at the same time (the delay $\delta$ is the same for all drivers).

3) We use the average distance traveled by the preceding vehicle to calculate the collision probabilities.

As in Case 1, the distance traveled by a vehicle until it completely stops if it does not collide is given by (3).

For $1 \leq i \leq N$, vehicle $C_{i}$ will collide with $C_{i-1}$ if and only if the distance needed by $C_{i}$ to stop is greater than the distance between them plus the average distance traveled by $C_{i-1}, \overline{l_{i-1}}$, so the collision probability of $C_{i}$ is:

$$
p_{i}=P\left(d_{s} \geq \overline{l_{i-1}}+s_{i}\right)=F\left(d_{s}-\overline{l_{i-1}}\right) .
$$

The average distance traveled by a vehicle, $\overline{l_{i}}$, must be computed recursively, starting from $\overline{l_{0}}=0$. For $1 \leq i \leq N$, the average distance traveled by vehicle $C_{i}$ is $\overline{l_{i}}=d_{s}(1-$ $\left.p_{i}\right)+d_{c, i} p_{i}$, where $d_{c, i}$ is the average distance traveled by the vehicle in case of collision:

$$
\begin{aligned}
d_{c, i} & =\frac{1}{p_{i}} \int_{0}^{d_{s}-\overline{l_{i-1}}}\left(\overline{l_{i-1}}+x\right) \lambda e^{-\lambda x} d x= \\
& =\frac{1}{p_{i}}\left(\overline{l_{i-1}}+\frac{1}{\lambda}-\left(d_{s}+\frac{1}{\lambda}\right) e^{-\lambda\left(d_{s}-\overline{l_{i-1}}\right)}\right) .
\end{aligned}
$$

Then, the equation for $\overline{l_{i}}$ is:

$$
\overline{l_{i}}= \begin{cases}d_{s}\left(1-p_{i}\right)+d_{c, i} p_{i}, & p_{i}>0 \\ d_{s}, & p_{i}=0 .\end{cases}
$$

Now, like in Case 1, we have to construct the matrix $\mathbf{P}$ and calculate the average number of accidents through (1).

3) Validation and discussion: Fig. 6 shows the results of computing the basic model described in the previous sections. The number of vehicles in the chain is $N=20$, and the rest of the parameters have been fixed at $a=8 \mathrm{~m} / \mathrm{s}^{2}$, which is the maximum deceleration of what is consider as a normal

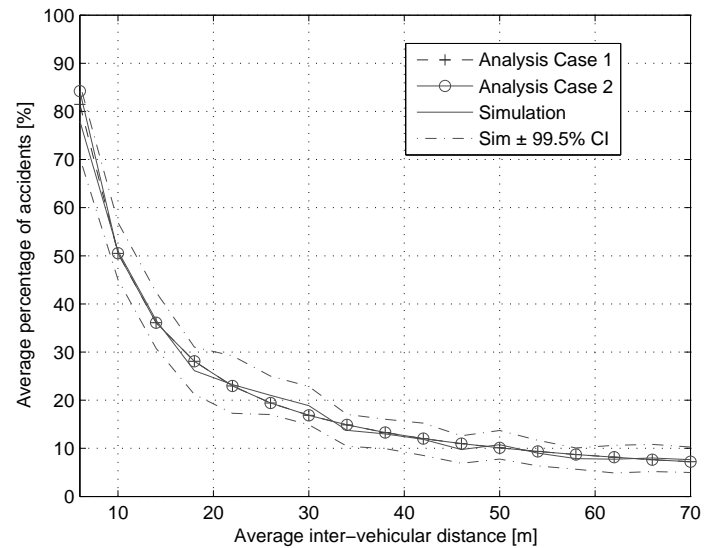

Fig. 6. Average percentage of accidents versus average inter-vehicle distance $\bar{s}=\frac{1}{\lambda+L} m$ for basic model, with exact solution (Case 1), approximate solution (Case 2) and Monte-Carlo simulation with a $99.5 \%$ confidence intervals.

vehicle [16], $V=33 \mathrm{~m} / \mathrm{s}$ and $\delta=T_{m, i}+T_{r, i}=0.1+0.9 \mathrm{~s}$. In this case, $T_{m, i}=0.1 s$ is the maximum delay for warning messages that vehicular communication standards specify [17], whereas $T_{r, i}=0.9 \mathrm{~s}$ is an average driver reaction time [18]. Fig. 6 illustrates the curves for the exact and the approximate basic models. In addition, a Monte-Carlo simulation of the system has been also conducted in order to validate our model. All the Monte-Carlo simulations in this paper have been performed with 10 replications per simulation point and results are shown with $99.5 \%$ confidence intervals. As can be seen using the average distance traveled by the preceding vehicle, $\overline{l_{i-1}}$, computed in Case 2, provides an excellent approximation to the exact collision probability, since the mean square error between the results of both cases is less than $0.5 \%$. Moreover, simulation results confirm that the model is correct enough, since the mean square error between the results of Case 2 and the Monte-Carlo simulation does not exceed $2 \%$.

\section{B. Influence of variability on deceleration, velocity and noti- fication delay}

In this section the basic model is extended by considering notification delays $\delta_{i}$, velocities $V_{i}$ and decelerations $a_{i}$ as variables. In most of the cases, they should be considered random variables with their appropriate probability density functions to model some particular effect. At this point, we do not assume any particular probability distribution for them. A discussion on this matter is provided later in Sect. IV-B1.

When deriving a model where all the involved parameters vary simultaneously, several problems arise. Our approach has been to derive a first model considering constant decelerations $a_{i}=a$, and then another one considering constant notification delays $\delta_{i}=\delta$. Later in this section this approach will be discussed and justified in detail.

Therefore let us first consider constant decelerations and variable velocities and notification delays. In this case, the distance needed to stop is not constant and equal for each 
vehicle anymore, but given by:

$$
d_{s, i}=\frac{V_{i}^{2}}{2 a}+V_{i} \delta_{i} .
$$

As in Case 1 , for $1 \leq i \leq N$ the collision probability is given by

$$
p_{i}=F\left(d_{s, i}-\overline{l_{i-1}}\right) .
$$

Again, the average distance traveled and the collision probabilities must be recursively computed. But in this case vehicle collisions may occur in four different ways: (1) vehicles $C_{i}$ and $C_{i-1}$ have not started to brake; (2) only one of them is braking; (3) both of them are braking; or (4) vehicle $C_{i-1}$ has stopped. Each one of these possibilities results in a different distance to stop, $d_{c_{1}, i}, d_{c_{2}, i}, d_{c_{3}, i}$ and $d_{c_{4}, i}$, that must be weighted by its probability of occurrence and added to get the average distance traveled $\overline{l_{i}}$ as:

$\overline{l_{i}}=d_{s, i}\left(1-p_{i}\right)+d_{c_{1}, i} q_{c_{1}, i}+d_{c_{2}, i} q_{c_{2}, i}+d_{c_{3}, i} q_{c_{3}, i}+d_{c_{4}, i} q_{c_{4}, i}$,

where

$d_{c_{1}, i}=\frac{1}{q_{c_{1}, i}} \int_{i n f_{1}}^{\text {sup }_{1}} V_{i} t_{c_{1}, i}(x) \lambda e^{-\lambda x} d x, \quad V_{i}>V_{i-1}$,

$d_{c_{2}, i}= \begin{cases}\frac{1}{q_{c_{2}, i}} \int_{\text {inf }_{2}}^{\text {sup }_{2}}\left(V_{i-1} t_{c_{2}, i}(x)+x\right) \lambda e^{-\lambda x} d x, & \delta_{i}<\delta_{i-1}, \\ \frac{1}{q_{c_{2}, i}} \int_{\text {inf }_{2}}^{\text {sup }_{2}} V_{i} t_{c_{2}, i}(x) \lambda e^{-\lambda x} d x, & \\ & \delta_{i}>\delta_{i-1},\end{cases}$

$d_{c_{3}, i}=\frac{1}{q_{c_{3}, i}} \int_{i n f_{3}}^{\text {sup }_{3}}\left(V_{i} t_{c_{3}, i}(x)-\frac{a}{2}\left(t_{c_{3}, i}-\delta_{i}\right)^{2}\right) \lambda e^{-\lambda x} d x$, $\left(V_{i}-V_{i-1}\right)+a\left(\delta_{i}-\delta_{i-1}\right) \neq 0,(16)$

$d_{c_{4}, i}=\frac{1}{q_{c_{4}, i}} \int_{i n f_{4}}^{s u p_{4}}\left(\overline{l_{i-1}}+x\right) \lambda e^{-\lambda x} d x$

and

$$
q_{c_{j}, i}=P\left(i n f_{j} \leq s_{i} \leq s u p_{j}\right)=F\left(\sup _{j}\right)-F\left(i n f_{j}\right),
$$

for $j=1, \ldots, 4$.

The functions $t_{c_{1}, i}(x), t_{c_{2}, i}(x)$ and $t_{c_{3}, i}(x)$ represent the time instants at which the collisions (1), (2) and (3) has occurred, where $x$ is the distance between $C_{i}$ and $C_{i-1}$. The derivation of these time functions as well as the above equations (14), (15), (16) and (17) and the appropriate values for the integration limits $i n f_{j}$ and $\sup _{j}$ for $j=1, \ldots, 4$ are provided in Appendix A. A discussion about the circumstances that cause the different ways of colliding is offered there as well.

At this point we can justify our previous discussed approach: if all the parameters are assumed variable, the number of possible ways in which collisions may occur increases remarkably, and all of them have to be taken into account for the computation of the average distance traveled by a vehicle, as $d_{c_{1}, i}, d_{c_{2}, i}, d_{c_{3}, i}$, and $d_{c_{4}, i}$ in (13). This fact makes the resulting equations cumbersome and hard to solve and makes it also difficult to describe the reasons why those events happen and to distinguish the influence of the different parameters on them. On the contrary, with our approach we can still obtain solutions for most of the cases by computing the solutions of the model for a range of the constant parameter. That is, we can plot a family of curves for the above model varying the deceleration and another family of curves for the next model varying the notification delay as shown later in Section V. Moreover, the first case with variable notification delay models a scenario where communications are in use but drivers have control over brake and so a driver reaction time has meaning and must be taken into consideration. The second case with constant notification delay exemplifies a scenario where communications are in use and the car is automatically braked as soon as a warning message is received.

Therefore, in the second step we consider $\delta_{i}$ constant. The distance to stop without collision of vehicle $C_{i}$ now includes a variable $a_{i}$ and becomes:

$$
d_{s, i}=\frac{V_{i}^{2}}{2 a_{i}}+V_{i} \delta .
$$

Using the same arguments as above, the collision probabilities are given by (12).

In this case collisions may occur only according to three different ways: (1) vehicles $C_{i}$ and $C_{i-1}$ have not started to brake; (2) both of them are already braking; or (3) vehicle $C_{i-1}$ has stopped, with their respective actual distances traveled, $d_{c_{1}, i}, d_{c_{2}, i}$ and $d_{c_{3}, i}$. Therefore, the average distance traveled by vehicle $C_{i}$ is given by

$$
\overline{l_{i}}=d_{s, i}\left(1-p_{i}\right)+d_{c_{1}, i} q_{c_{1}, i}+d_{c_{2}, i} q_{c_{2}, i}+d_{c_{3}, i} q_{c_{3}, i},
$$

where $q_{c_{1}, i}, q_{c_{2}, i}$ and $q_{c_{3}, i}$ are given by (18), $d_{c_{1}, i}$ and $d_{c_{3}, i}$ have the same form of (14) and (17) respectively, with slightly different integration limits and time functions which are derived in Appendix $\mathrm{B}$, and $d_{c_{2}, i}$ is expressed by:

$$
d_{c_{2}, i}=\left\{\begin{array}{c}
\frac{1}{q_{c_{2}, i}} \int_{i n f_{2}}^{\sup _{2}}\left(V_{i} t_{c_{2}, i}(x)-\right. \\
\left.\quad-\frac{a_{i}}{2}\left(t_{c_{2}, i}(x)-\delta\right)^{2}\right) \lambda e^{-\lambda x} d x \\
V_{i}>V_{i-1}, a_{i-1}-a_{i}=0 \\
\frac{1}{q_{c_{2 a}, i}} \int_{i n f_{2 a}}^{s u p_{2 a}}\left(V_{i} t 1_{c_{2}, i}(x)-\right. \\
\left.-\frac{a_{i}}{2}\left(t 1_{c_{2}, i}(x)-\delta\right)^{2}\right) \lambda e^{-\lambda x} d x+ \\
\quad+\frac{1}{q_{c_{2 b}, i}} \int_{i n f_{2 b}}^{s u p_{2 b}}\left(V_{i} t 2_{c_{2}, i}(x)-\right. \\
\left.-\frac{a_{i}}{2}\left(t 2_{c_{2}, i}(x)-\delta\right)^{2}\right) \lambda e^{-\lambda x} d x \\
\quad a_{i-1}-a_{i} \neq 0 .
\end{array}\right.
$$

Note that in all the cases the above equations provide additional meaningful information since we have derived the probability of the different ways in which vehicle collisions may occur $\left(q_{c_{j}, i}\right)$ as well as the average distance traveled by the vehicles $\left(d_{c_{j}, i}\right)$. This information can be used, for instance, 
to design a measure about the severity of collisions that assigns different weights to every particular type of collision. Finally, one may wonder whether it is possible to adopt a simpler approximation, instead of the average distance $\overline{l_{i}}$ to compute the probabilities, or whether there is significant difference between the $\overline{l_{i}}$. Indeed, for vehicles located in the chain far enough from the leading vehicle, $\overline{l_{i}}$ are closer to eq. (2), that is, the influence of the outcome of the previous vehicle is weak and the outcome depends mainly on the particular values of the parameters. However, for vehicles close to the leading vehicle, there is a strong dependence on the outcome of the previous vehicle and eqs. (13) and (20) cannot be neglected.

1) Validation and discussion: The next stage would be to assign the kinematic parameters and notification delays appropriate values that model realistic scenarios. As an example, in order to take into account an underlying communication model, the notification delay should be assumed to be a random variable with an appropriate probability density function. In this way, information packet collisions in a heavily loaded shared communications channel can be modeled with an appropriate random variable for the access delay and characterized also by $T_{m, i}$ [11]. Furthermore, since vehicles move at different speeds, the velocity should be assumed to be a random variable too. Let us note that, in most of the practical cases, inter-vehicle distances and velocities represent the state of the system when the incident occurs, and so they should be considered random variables, though determining their distributions and ranges require a proper characterization of the scenario of interest. Accelerations and delays can be controlled by different means after the incident, and so depending on the application evaluated they can be considered constant or assigned particular values.

However, with regard to the analysis, introducing additional random variables makes it hard to obtain a closedform solution for the collision probabilities, even for the simple case when parameters are assigned uniform distributed random variables, and the benefits are not clear. Therefore, the solutions when a parameter is a random variable have been computed numerically. The parameters are supposed to be uniform random variables and eq. (1) has been computed 100 times and averaged. In all the cases we assume a chain of $N=20$ vehicles.

A solution for the model with constant deceleration has been computed for three different scenarios. In the first one, $\delta_{i}$ is assumed to be a uniform random variable ranging between 0.5 and $1.5 \mathrm{~s}$, whereas the velocity has been fixed at $V=33 \mathrm{~m} / \mathrm{s}$. In the second scenario, $V_{i}$ is assumed to be a uniform random variable between 30 and $36 \mathrm{~m} / \mathrm{s}$ and the notification delay has been fixed at $\delta=1 \mathrm{~s}$. In the last simulation, both the velocity and the notification delay are assumed to be uniform random variables ranging between 0.5 and $1.5 s$ and between 30 and $36 \mathrm{~m} / \mathrm{s}$, respectively. In all the simulations the deceleration is kept constant at $8 \mathrm{~m} / \mathrm{s}^{2}$.

The validation of the model with constant notification delay has been also done for three different scenarios. In the first one, deceleration $a_{i}$ is assumed to be a uniform random variable between 4 and $8 \mathrm{~m} / \mathrm{s}^{2}$, whereas the velocity has been fixed at

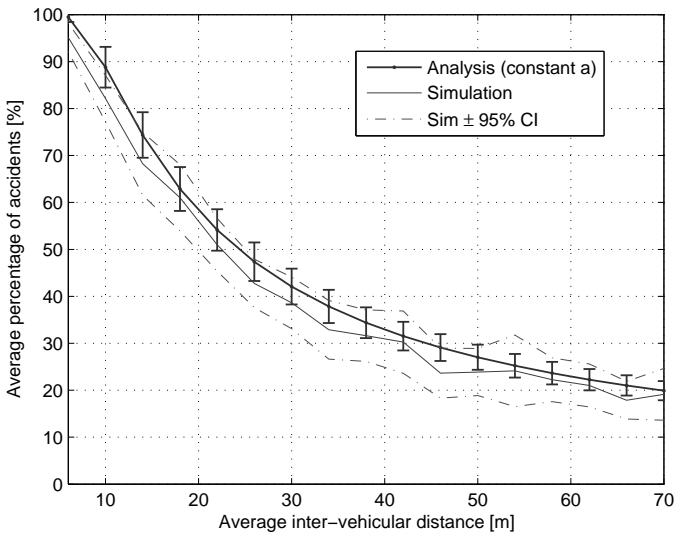

(a) $\delta_{i} \sim U(0.5,1.5) \mathrm{s}, V_{i}=33 \mathrm{~m} / \mathrm{s}$ and $a_{i}=8 \mathrm{~m} / \mathrm{s}^{2}$.

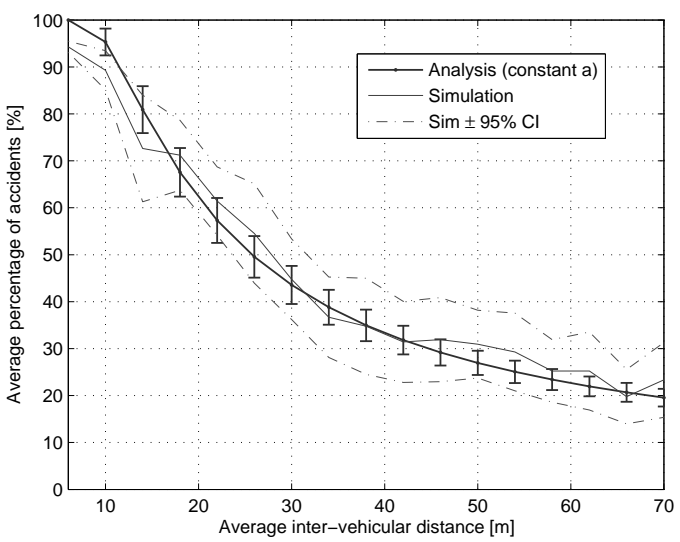

(b) $\delta_{i}=1 \mathrm{~s}, V_{i} \sim U(30,36) \mathrm{m} / \mathrm{s}$ and $a_{i}=8 \mathrm{~m} / \mathrm{s}^{2}$.

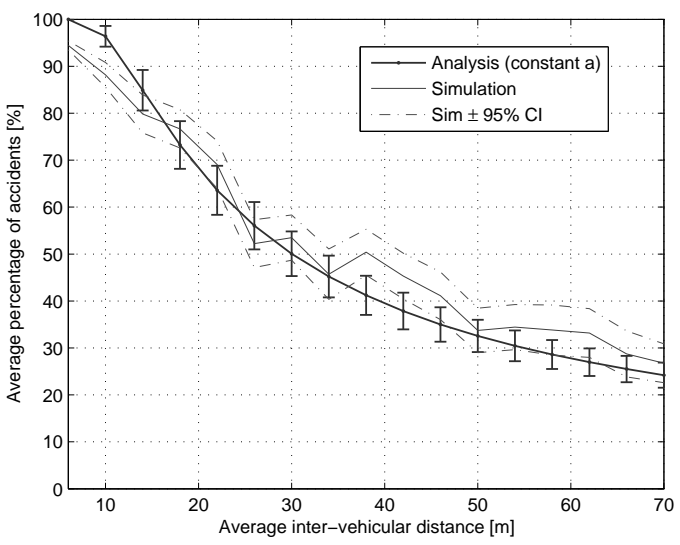

(c) $\delta_{i} \sim U(0.5,1.5) \mathrm{s}, V_{i} \sim U(30,36) \mathrm{m} / \mathrm{s}$ and $a_{i}=8 \mathrm{~m} / \mathrm{s}^{2}$.

Fig. 7. Validation of the model with constant deceleration through the evaluation of three different scenarios.

$V=33 \mathrm{~m} / \mathrm{s}$. In the second one, $V_{i}$ is assumed to be a uniform random variable between 30 and $36 \mathrm{~m} / \mathrm{s}$ and the deceleration has been fixed at $a=8 \mathrm{~m} / \mathrm{s}^{2}$. In the last simulation, both the deceleration and the velocity are assumed to be uniform random variables between 4 and $8 \mathrm{~m} / \mathrm{s}^{2}$ and between 30 and $36 \mathrm{~m} / \mathrm{s}$, respectively. In all the simulations the notification delay is kept constant at $1 \mathrm{~s}$.

Finally, in order to validate the results for our solutions, the 


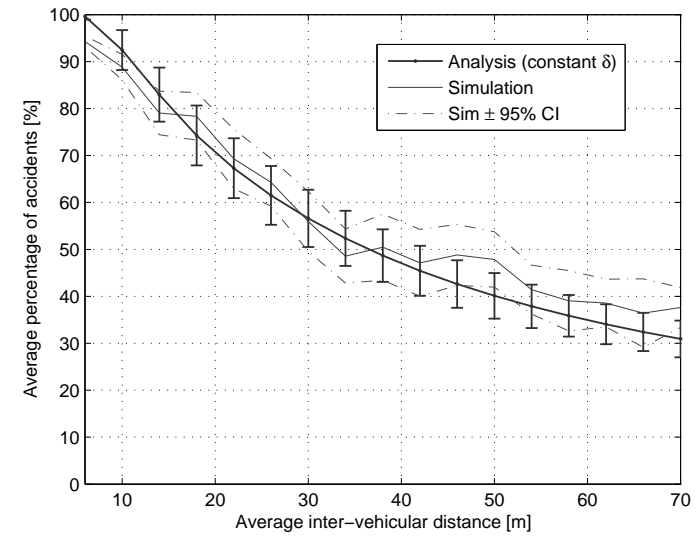

(a) $\delta_{i}=1 \mathrm{~s}, V_{i}=33 \mathrm{~m} / \mathrm{s}$ and $a_{i} \sim U(4,8) \mathrm{m} / \mathrm{s}^{2}$.

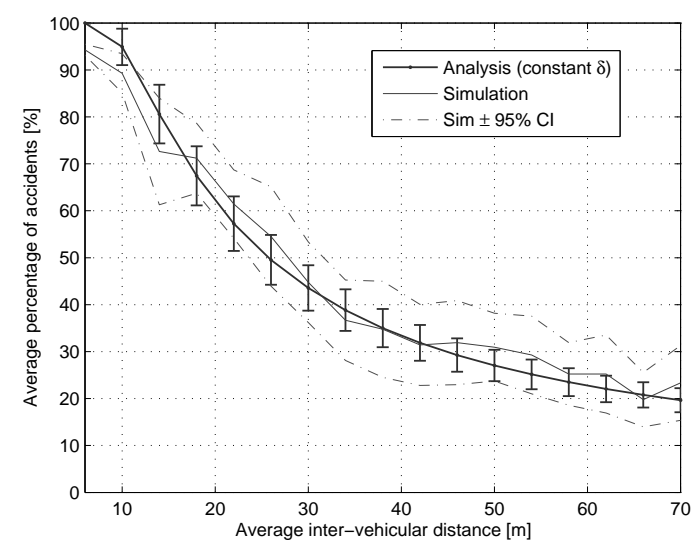

(b) $\delta_{i}=1 s, V_{i} \sim U(30,36) \mathrm{m} / \mathrm{s}$ and $a_{i}=8 \mathrm{~m} / \mathrm{s}^{2}$.

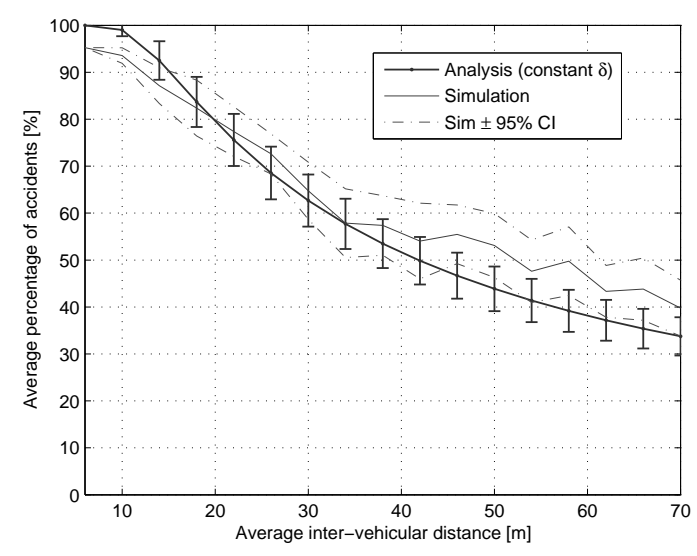

(c) $\delta_{i}=1 \mathrm{~s}, V_{i} \sim U(30,36) \mathrm{m} / \mathrm{s}$ and $a_{i} \sim U(4,8) \mathrm{m} / \mathrm{s}^{2}$.

Fig. 8. Validation of the model with constant notification delay through the evaluation of three different scenarios.

corresponding Monte-Carlo simulations have been conducted as well.

Figures 7 and 8 show the results of this section. Let us remark that these pictures are provided to validate that our model shows correctly the dynamics of the system. A discussion on the influence of the parameters on the collision process is deferred to the next Section. The average number of accidents computed with our model for each of the six cases is compared with the aforementioned Monte-Carlo simulations. The standard deviation has been computed and shown as errorbars. Dashed lines show the $95 \%$ confidence interval of the corresponding simulation. In all the cases, the results reasonably confirm the validity of our model, even using $\overline{l_{i-1}}$ as approximation, since the mean square error between the results of the analysis and the simulation remains between $3.5 \%$ and $6 \%$ for all the cases.

\section{APPlications AND Discussion OF THE MODEL}

Once our model has been validated in the previous sections, we use it to evaluate the influence of the different parameters on the vehicle collision process. In this section we present some results as an example of the utility of our model. The metric used here is the average percentage of accidents in the chain, but the model could also provide information about the probability of collision or the average distance traveled for the different manners a collision can occur. A systematic evaluation of different scenarios as well as different metrics is left as future work. First, we focus on qualitative aspects of the influence of the parameters on the collision process that our model can quickly reveal. Then, we discuss quantitative aspects of the results provided in this section.

As for the qualitative evaluation, we first provide a set of figures that show the influence of the different parameters. Fig. 9 shows a family of curves for both instances of the model over a range of their constant parameter, $a$ or $\delta$. As can be seen in Fig. 9(a), the number of accidents is clearly sensitive to the deceleration capabilities of the vehicles, which agrees with the results obtained in [2]. However, it does not seem to be statistical difference for different notification delays when the deceleration and velocities are variable. This result is also in accordance with [2], where it is shown that moderate changes in the notification delay cause small variations in accident severity. Later in this Section we discuss when the delay actually has an important influence on the number of accidents.

Fig. 10(a) shows the results when the velocities are randomly distributed. In this case if either deceleration or notification delay are kept constant it causes a reduction of the number of accidents. In fact, in this case it is noticeable the positive effect of a communication system able to deliver warning messages with short maximum delays and automatic vehicle response. Fig. 10(b) shows similar results when deceleration is kept constant at $a=6 \mathrm{~m} / \mathrm{s}^{2}$. The results however reveal that in general the variability of the kinetic parameters has a negative impact on the number of accidents. If the system is able to keep constant some of the parameters during the emergency event, an improvement can be achieved. The benefits of a warning collision system are even clearer in Fig. 10(c). When all the parameters remain constant, a shorter notification delay always results in fewer vehicle accidents.

Overall, these results suggest that a cooperative warning collision notification system combined with a vehicle control system able to smooth out the variations of speed and deceleration of the platoon of vehicles may improve the driver and passengers safety. In fact, more detailed conclusions can be 


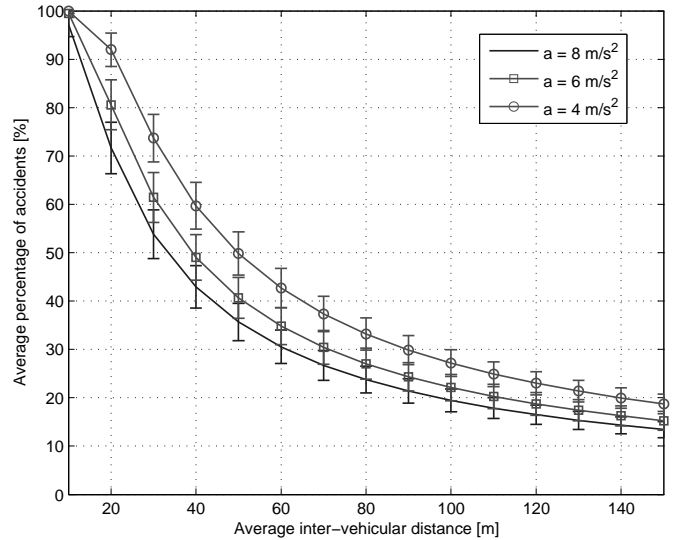

(a) Average percentage of accidents for different decelerations when $V_{i} \sim U(30,36) \mathrm{m} / \mathrm{s}$ and $\delta_{i} \sim U(0.5,1.8) s$.

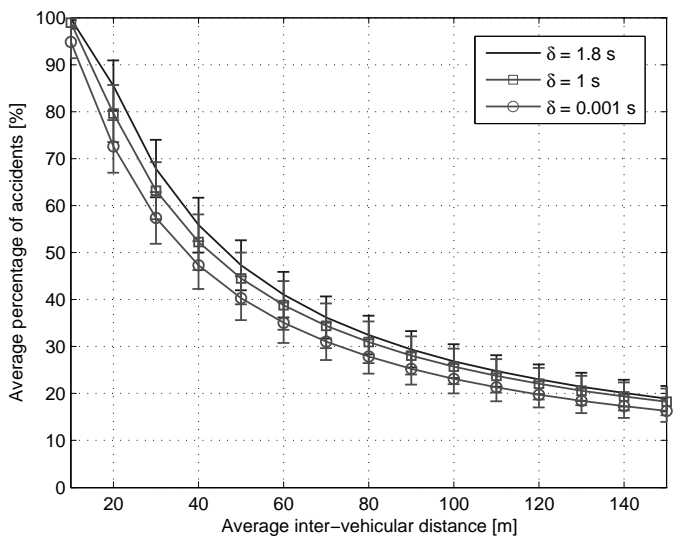

(b) Average percentage of accidents for different notification delay when $V_{i} \sim U(30,36) \mathrm{m} / \mathrm{s}$ and $a_{i} \sim U(4,8) \mathrm{m} / \mathrm{s}^{2}$.

Fig. 9. Performance of the model with different constant decelerations (a) and message reception delay (b).

extracted. As we said in the introduction, our model is useful to provide general guidelines about the design and operation of a CCA application. For instance, a CCA application may be based on a warning message delivered by an appropriate Human Machine Interface (HMI) [19], a specific sound for instance as in [20], or by a fully automated braking response [21], [22]. The latter is expected to provide better performance, but one may wonder if the former benefits from a communication system and for which range of parameters. Now, how can our model be applied to obtain relevant conclusions about these questions? First, it must be taken into account that for a reactive CCA application, the only parameters in our model that can be controlled are delays, with the communication system, and decelerations, with some automated control response to the warning message. Let us recall that either the velocities of the vehicles and the inter-vehicle distance model the state of the traffic when the incident occurs and so both of them should be considered a random variable. Therefore, in the best case, the CCA is able to provide a constant and short delay and enforce an appropriate constant deceleration. So, the curve in Fig. 10(b), provides the results for this case. If there is

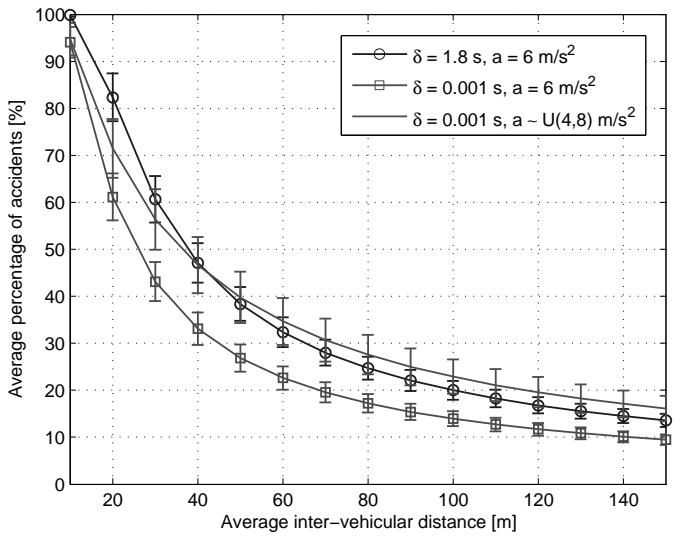

(a) Variability of deceleration with different notification delays.

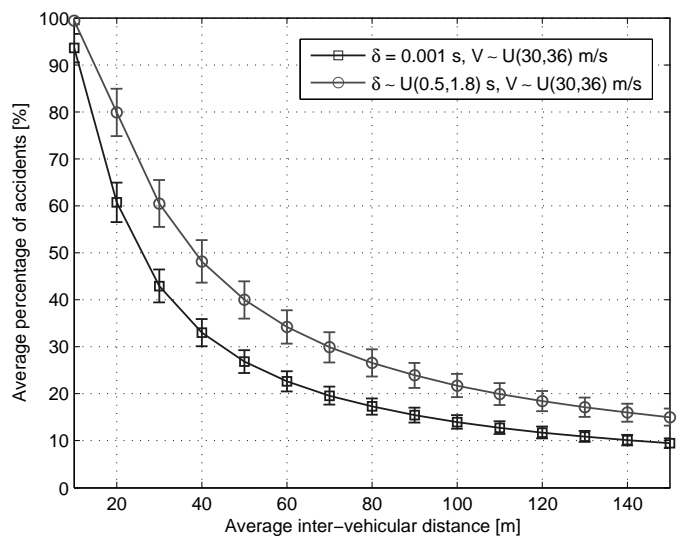

(b) Variability of velocity and notification delay.

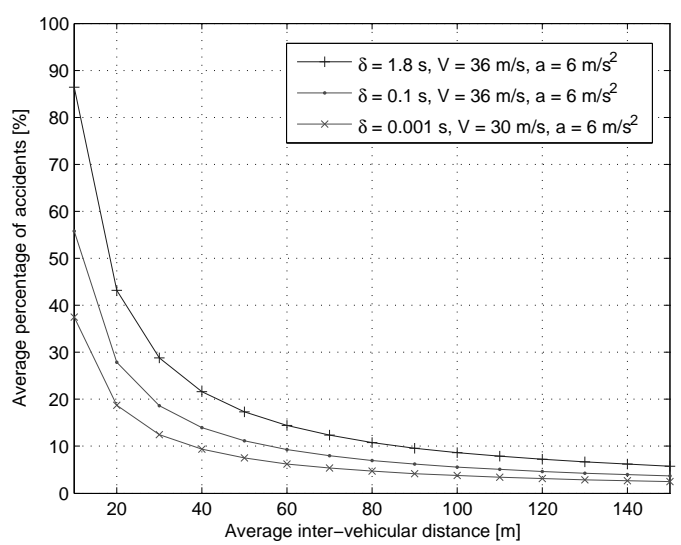

(c) Performance of the model with fixed parameters.

Fig. 10. Evaluation of the impact of the parameters' variability on the number of vehicle collisions.

a warning message, but the driver still keeps the control of braking, a random reaction time should be added before the brake. This case is provided by the curve with uniform delay again in Fig. 10(c), assuming that the deceleration can be kept constant, which is not quite realistic. It is more reasonable that every driver applies also a different deceleration, which is the case shown in Fig. 9(b). But, as shown, in this case the actual delay is of little relevance. This has important 


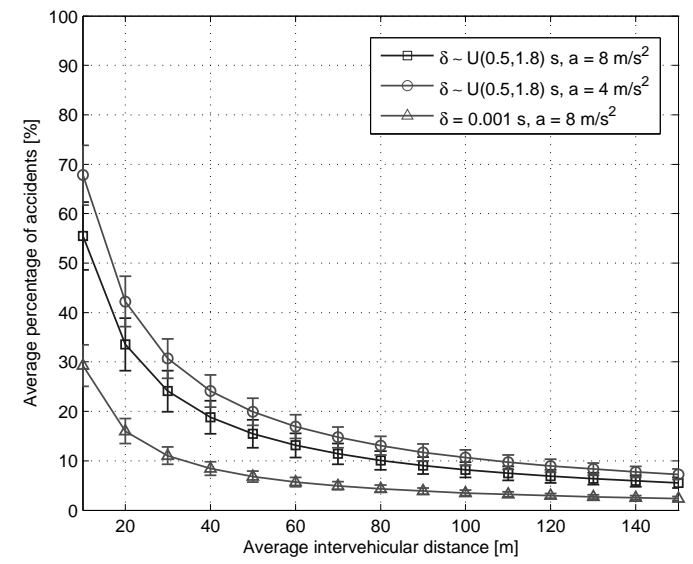

Fig. 11. Average percentage of accidents in a low speed scenario with $V_{i} \sim U(10,16) \mathrm{m} / \mathrm{s}$.

implications in the design of the CCA. The usual approach is to consider that the emergency messages must be sent as fast as possible [15], [23], but according to these results a higher delay could be traded off for other features such as reliability of warning message reception. For instance, adding a RequestTo-Send/Clear-To-Send mechanism to avoid packet collisions due to hidden nodes [1]. Or more importantly, the CCA application should provide an acceleration control mechanism, so the margin in delay can be used to collect all the necessary information from neighbor vehicles to perform such control properly. This kind of insights on delay requirements is also important for designing CCA applications based on predicting trajectory conflicts, in order to determine the time horizon for trajectory estimation [3].

However, if we consider a low speed and high density scenario, the delay has a remarkable influence. Fig. 11 shows the average percentage of accidents when velocities are uniformly distributed within 10 and $16 \mathrm{~m} / \mathrm{s}$. This scenario would model a urban road, where speed is relatively low but the vehicle density is high $^{3}$. And in this case, specially at short intervehicle distances corresponding to urban roads, the influence of delay is more noticeable, higher than that of deceleration. Therefore we can conclude that the use of an HMI message might not be sufficient to ensure safety and a special emphasis should be placed on providing automatic deceleration control. Moreover, in this scenario it is specially difficult for a communication system based on contention channel access (CSMA) to provide low delays, since the number of neighbors in range is high, unless additional congestion control mechanisms such as transmit power control are applied.

In fact, some of these conclusions can be drawn by directly examining eq. (12), that is, for high speeds it is more important to have good deceleration capabilities rather than to press the brake quickly, and conversely for low speeds. The previous discussion is provided to show that the model has potential to provide interesting qualitative and quantitative conclusions

\footnotetext{
${ }^{3}$ Just for the sake of example, but let us remark that a log-normal distribution for inter-vehicle distances describes more accurately high density scenarios.
}

about the colliding process and the mechanisms that CCA applications should include, but we have to remark that a precise definition of the scenarios of interest is still necessary, to set appropriate distributions and ranges for the parameters, which is left as future work.

Finally, as for the quantitative aspects of the results, the percentage of accidents might seem higher than expected, above $10 \%$ in many cases, as well as the slow decay of it for high inter-vehicular distances. This is first a consequence of the extreme case we are evaluating here, that is, the leading vehicle stops completely and immediately. It makes the collision of the first car of the platton almost unavoidable in most of the cases. As a worst case approach, better outcomes are expected in reality. But also these results have to be interpreted with care, since using average inter-vehicle distances may lead to misleading conclusions. As an example, with the parameters used in Fig. 10(c), $V=36 \mathrm{~m} / \mathrm{s}, a=6 \mathrm{~m} / \mathrm{s}^{2}$ and $\delta=0.1$, the distance needed to stop is $111.6 \mathrm{~m}$. For an exponentially distributed inter-vehicle distance with mean $\bar{s}=60 \mathrm{~m}$, the probability of $s_{i}$ being less than $100 \mathrm{~m}$ is 0.81 , and even with a mean $\bar{s}=150 \mathrm{~m}$, this probability is still 0.48 . So the probability of collision is higher than one may intuitively think, specially for the first vehicles in the chain. Therefore, even at relatively high inter-vehicular distances, the collisions are mainly suffered by the first and second vehicle, which accounts for the $10 \%$ of accidents for our example with $N=20$ vehicles.

\section{CONCLUSIONS}

In this paper we propose and derive a stochastic model for the probability of collisions in a chain of vehicles where a warning collision system is in operation. The fact that a warning notification system is used allows us to overcome the difficulties for obtaining stochastic models for such vehicular scenarios, since we can assume that all the drivers/vehicles react to the warning message independently, and therefore the motion equations can be simplified. We also propose a good matching approximation to the exact model to further reduce the required computations to calculate the vehicle collision probabilities. In both cases, its validity has been confirmed by Monte-Carlo simulations.

The model is independent of the particular communication system employed as long as its operation can be abstracted and characterized by an appropriate message notification delay including communication latency and driver reaction times. Therefore, it also enables the performance evaluation of different technologies. Indeed, a future line of this work is to assess the performance of current VANET technology based on contention (CSMA) MAC protocols for those cases where delay is actually relevant for the collision process outcome. Similarly, different probability distributions for the inter-vehicular spacing can be incorporated seamlessly into the model, due to the fact that the distribution of the initial intervehicle spacing is independent of the actions that drivers make after receiving the warning messages. Here we have used an exponential distribution, which is considered appropriate for low vehicle traffic densities. As a future work we plan to 
employ a log-normal distribution which describes well high vehicle traffic densities. Finally, we compute the probability that collisions occur in different forms (both vehicles in motion, one stopped and one in motion, etc.), which opens a promising way to define detailed accident severity functions, that is, by assigning different grades of severity to each collision possibility. This is an interesting approach that we leave as future work as well.

Although we have shown some examples of the application of the model, a quantitative evaluation requires a careful definition of the scenarios of interest. Therefore, we leave as future but imminent direction to pursue a systematic characterization and evaluation of different scenarios for a wider and more accurate extent of the model parameters.

\section{APPENDiX A. COMPUTATION OF THE DisTANCE TRAVELED BY A VEHICLE FOR VARIABLE VELOCITY AND NOTIFICATION DELAY}

Let us recall that when velocity and notification delay are not constant the collisions may occur in four different ways: (1) vehicles $C_{i}$ and $C_{i-1}$ have not started to brake; (2) only one of them is braking; (3) both of them are braking; or (4) vehicle $C_{i-1}$ has stopped. Each one of these possibilities results in a different distance to stop, $d_{c_{1}, i}, d_{c_{2}, i}, d_{c_{3}, i}$ and $d_{c_{4}, i}$ respectively that must be weighted by the probability of the event and added to get the average $\bar{l}_{i}$ as in (13).

The computation of these distance is as follows:

(1) Collision when the vehicles have not started to brake $\left(d_{c_{1}, i}\right)$. This event may happen if the difference of initial velocities makes the vehicles crash before receiving the warning message. $^{4}$

A time instant $t_{c_{1}, i}\left(s_{i}\right)$ should exist so that

$$
\begin{gathered}
V_{i} t_{c_{1}, i}\left(s_{i}\right)=V_{i-1} t_{c_{1}, i}\left(s_{i}\right)+s_{i}, \\
0 \leq t_{c_{1}, i}\left(s_{i}\right) \leq \min \left\{\delta_{i}, \delta_{i-1}\right\} .
\end{gathered}
$$

Solving (22) we obtain

$$
t_{c_{1}, i}\left(s_{i}\right)=\frac{s_{i}}{V_{i}-V_{i-1}}
$$

Therefore, the distance to stop in this case is $d=V_{i} t_{c_{1}, i}\left(s_{i}\right)$, which is a function of $s_{i}$ (exponentially distributed). Then, the average distance is computed as follows:

$$
d_{c_{1}, i}=\frac{1}{F\left(\text { sup }_{1}\right)-F\left(i n f_{1}\right)} \int_{i n f_{1}}^{\sup _{1}} \frac{V_{i} x}{V_{i}-V_{i-1}} \lambda e^{-\lambda x} d x .
$$

Now, it remains to compute the range of $s_{i}$ where a collision can happen, that is, the integration limits denoted as in $f_{1}$ and sup $_{1}$ :

${ }^{4}$ Let us note that this case implies that the vehicles would collide even if there is no obstacle ahead on the road or the rest of vehicles in the chain are not braking. For instance, a driver notices that his actual speed is higher than that of the preceding vehicle but does not reduce it and let his car collide. One might think of a situation where bad weather conditions, like a very thick fog, prevent the driver from noticing the risk. In a normal driving situation this case should be highly unlikely but it has to be considered to get a consistent result.
- If $V_{i}-V_{i-1} \leq 0$, there is no solution (no collision can occur). Let us define appropriate limits

$$
\begin{aligned}
& \text { in } f_{1}=0, \\
& \sup _{1}=0 .
\end{aligned}
$$

- If $V_{i}-V_{i-1}>0$, then (23) holds if and only if $0 \leq s_{i} \leq$ $\left(V_{i}-V_{i-1}\right) \cdot \min \left\{\delta_{i}, \delta_{i-1}\right\}$. Let us define

$$
\begin{aligned}
& \text { inf }_{1}=0, \\
& \sup _{1}=\left(V_{i}-V_{i-1}\right) \cdot \min \left\{\delta_{i}, \delta_{i-1}\right\} .
\end{aligned}
$$

The same procedure is applied for the following cases: the computation of the actual distance traveled, in this case $d=V_{i} t_{c_{1}, i}\left(s_{i}\right)$, where $t_{c_{1}, i}\left(s_{i}\right)=f\left(s_{i}, a, V_{i}, V_{i-1}, \delta_{i}, \delta_{i-1}\right)$, which is multiplied by the exponential pdf and integrated within the appropriate limits, which are also derived.

(2) Collision when only one vehicle is braking $\left(d_{c_{2}, i}\right)$. In this case, the collision event depends on the relative reaction times of the drivers. That is, due to a high reaction time, one of the drivers starts to brake too late.

- If $\delta_{i}=\delta_{i-1}$, then we have to skip to case (3), later in the text, and so let us define

$$
\begin{aligned}
& \text { inf }_{2}=\sup _{1}, \\
& \text { sup }_{2}=\sup _{1} .
\end{aligned}
$$

- If $\delta_{i}<\delta_{i-1}$, then vehicle $C_{i}$ starts to brake before $C_{i-1}$ does.

A time instant $t_{c_{2}, i}\left(s_{i}\right)$ should exist so that

$$
\begin{aligned}
& V_{i} t_{c_{2}, i}\left(s_{i}\right)-\frac{a}{2}\left(t_{c_{2}, i}\left(s_{i}\right)-\delta_{i}\right)^{2}=V_{i-1} t_{c_{2}, i}\left(s_{i}\right)+s_{i}, \\
& \delta_{i} \leq t_{c_{2}, i}\left(s_{i}\right) \leq \delta_{i-1}
\end{aligned}
$$

Solving this equation, we obtain the following solutions:

$$
\begin{aligned}
t 1_{c_{2}, i}\left(s_{i}\right) & =\frac{V_{i}-V_{i-1}+\delta_{i}-}{a} \\
& -\sqrt{\left(\frac{V_{i}-V_{i-1}}{a}+\delta_{i}\right)^{2}-\delta_{i}^{2}-\frac{2 s_{i}}{a}}, \\
t 2_{c_{2}, i}\left(s_{i}\right) & =\frac{V_{i}-V_{i-1}+\delta_{i}+}{a} \\
& +\sqrt{\left(\frac{V_{i}-V_{i-1}}{a}+\delta_{i}\right)^{2}-\delta_{i}^{2}-\frac{2 s_{i}}{a}} .
\end{aligned}
$$

The term in the square root is positive if and only if $s_{i} \leq \delta_{i}\left(V_{i}-V_{i-1}\right)+\frac{\left(V_{i}-V_{i-1}\right)^{2}}{2 a}$.

It can be proved that (33) does not hold for $t 2_{c_{2}, i}\left(s_{i}\right)$, so the only possible solution is $t 1_{c_{2}, i}\left(s_{i}\right)$.

- If $V_{i}-V_{i-1}>a\left(\delta_{i-1}-\delta_{i}\right)$, then (33) holds for $t 1_{c_{2}, i}\left(s_{i}\right)$ if and only if

$$
\delta_{i}\left(V_{i}-V_{i-1}\right) \leq s_{i} \leq \delta_{i-1}\left(V_{i}-V_{i-1}\right)-\frac{a}{2}\left(\delta_{i}-\delta_{i-1}\right)^{2} .
$$


Let us define

$$
\begin{aligned}
\inf _{2}= & \delta_{i}\left(V_{i}-V_{i-1}\right), \\
\sup _{2}= & \min \left\{\delta_{i}\left(V_{i}-V_{i-1}\right)+\frac{\left(V_{i}-V_{i-1}\right)^{2}}{2 a},\right. \\
& \left.\delta_{i-1}\left(V_{i}-V_{i-1}\right)-\frac{a}{2}\left(\delta_{i}-\delta_{i-1}\right)^{2}\right\} .
\end{aligned}
$$

- If $0<V_{i}-V_{i-1} \leq a\left(\delta_{i-1}-\delta_{i}\right)$, then (33) holds for $t 1_{c_{2}, i}\left(s_{i}\right)$ if and only if

$\delta_{i}\left(V_{i}-V_{i-1}\right) \leq s_{i} \leq \delta_{i}\left(V_{i}-V_{i-1}\right)+\frac{\left(V_{i}-V_{i-1}\right)^{2}}{2 a}$.

Let us define

$$
\begin{aligned}
& \inf _{2}=\delta_{i}\left(V_{i}-V_{i-1}\right), \\
& \sup _{2}=\delta_{i}\left(V_{i}-V_{i-1}\right)+\frac{\left(V_{i}-V_{i-1}\right)^{2}}{2 a} .
\end{aligned}
$$

- Otherwise, there is no solution, and so let us define

$$
\begin{aligned}
& \text { inf }_{2}=\text { sup }_{1}, \\
& \text { sup }_{2}=\text { sup }_{1} .
\end{aligned}
$$

- If $\delta_{i}>\delta_{i-1}$, then vehicle $C_{i-1}$ starts to brake before $C_{i}$ does.

A time instant $t_{c_{2}, i}\left(s_{i}\right)$ should exist so that

$$
\begin{aligned}
& V_{i} t_{c_{2}, i}\left(s_{i}\right)=V_{i-1} t_{c_{2}, i}\left(s_{i}\right)-\frac{a}{2}\left(t_{c_{2}, i}\left(s_{i}\right)-\delta_{i-1}\right)^{2}+s_{i}, \\
& \delta_{i-1} \leq t_{c_{2}, i}\left(s_{i}\right) \leq \delta_{i} .
\end{aligned}
$$

Solving (44), we obtain the following solutions:

$$
\begin{aligned}
& t 1_{c_{2}, i}\left(s_{i}\right)=\frac{V_{i-1}-V_{i}}{a}+\delta_{i-1}- \\
&-\sqrt{\left(\frac{V_{i-1}-V_{i}}{a}+\delta_{i-1}\right)^{2}-\delta_{i-1}^{2}+\frac{2 s_{i}}{a}} \\
& t 2_{c_{2}, i}\left(s_{i}\right)=\frac{V_{i-1}-V_{i}}{a}+\delta_{i-1}+ \\
&+\sqrt{\left(\frac{V_{i-1}-V_{i}}{a}+\delta_{i-1}\right)^{2}-\delta_{i-1}^{2}+\frac{2 s_{i}}{a}} .
\end{aligned}
$$

The term in the square root is positive if and only if $s_{i} \geq \delta_{i-1}\left(V_{i}-V_{i-1}\right)-\frac{\left(V_{i-1}-V_{i}\right)^{2}}{2 a}$.

It can be proved that (45) does not hold for $t 1_{c_{2}, i}\left(s_{i}\right)$, so the only possible solution is $t 2_{c_{2}, i}\left(s_{i}\right)$.

- If $V_{i-1}-V_{i}<0$, then (45) holds for $t 2_{c_{2}, i}\left(s_{i}\right)$ if and only if

$$
\delta_{i-1}\left(V_{i}-V_{i-1}\right) \leq s_{i} \leq \delta_{i}\left(V_{i}-V_{i-1}\right)+\frac{a}{2}\left(\delta_{i}-\delta_{i-1}\right)^{2} .
$$

Let us define

$$
\begin{aligned}
& \text { inf }_{2}=\delta_{i-1}\left(V_{i}-V_{i-1}\right), \\
& \sup _{2}=\delta_{i}\left(V_{i}-V_{i-1}\right)+\frac{a}{2}\left(\delta_{i}-\delta_{i-1}\right)^{2} .
\end{aligned}
$$

- If $0 \leq V_{i-1}-V_{i} \leq a\left(\delta_{i}-\delta_{i-1}\right)$, then (45) holds for $t 2_{c_{2}, i}\left(s_{i}\right)$ if and only if

$$
\begin{array}{r}
\delta_{i-1}\left(V_{i}-V_{i-1}\right)-\frac{\left(V_{i-1}-V_{i}\right)^{2}}{2 a} \leq s_{i} \leq \\
\leq \delta_{i}\left(V_{i}-V_{i-1}\right)+\frac{a}{2}\left(\delta_{i}-\delta_{i-1}\right)^{2} .
\end{array}
$$

Let us define

$$
\begin{aligned}
& \text { inf }_{2}=\delta_{i-1}\left(V_{i}-V_{i-1}\right)-\frac{\left(V_{i-1}-V_{i}\right)^{2}}{2 a}, \\
& \sup _{2}=\delta_{i}\left(V_{i}-V_{i-1}\right)+\frac{a}{2}\left(\delta_{i}-\delta_{i-1}\right)^{2} .
\end{aligned}
$$

- Otherwise, there is no solution, and so let us define

$$
\begin{aligned}
& \text { inf }_{2}=\sup _{1}, \\
& \sup _{2}=\sup _{1} .
\end{aligned}
$$

(3) Collision when both vehicles are braking $\left(d_{c_{3}, i}\right)$. In this case, both vehicles are aware of the danger and have started to brake but they are not able to avoid the collision, due to their initial speeds and reaction times, and they collide in motion.

A time instant should exist so that

$$
\begin{aligned}
& V_{i} t_{c_{3}, i}\left(s_{i}\right)-\frac{a}{2}\left(t_{c_{3}, i}\left(s_{i}\right)-\delta_{i}\right)^{2}= \\
& =V_{i-1} t_{c_{3}, i}\left(s_{i}\right)-\frac{a}{2}\left(t_{c_{3}, i}\left(s_{i}\right)-\delta_{i-1}\right)^{2}+s_{i}, \\
& \delta_{i} \leq t_{c_{3}, i}\left(s_{i}\right) \leq \frac{V_{i}}{a}+\delta_{i}, \\
& \delta_{i-1} \leq t_{c_{3}, i}\left(s_{i}\right) \leq T_{i-1}\left(\overline{l_{i-1}}\right)
\end{aligned}
$$

where $T_{i-1}\left(\overline{l_{i-1}}\right)$ is the time needed by vehicle $C_{i-1}$ to travel the distance $\overline{l_{i-1}}$, and it is calculated by the function:

$$
T_{i}(x)= \begin{cases}\frac{x}{V_{i}}, & \text { if } x \leq V_{i} \delta_{i}, \\ \frac{V_{i}}{a}+\delta_{i}-\sqrt{\frac{2}{a}\left(d_{s, i}-x\right)}, & \text { if } x>V_{i} \delta_{i} .\end{cases}
$$

Solving (56), we obtain

$$
t_{c_{3}, i}\left(s_{i}\right)=\frac{s_{i}+\frac{a}{2}\left(\delta_{i}^{2}-\delta_{i-1}^{2}\right)}{\left(V_{i}-V_{i-1}\right)+a\left(\delta_{i}-\delta_{i-1}\right)} .
$$

In order to simplify the notation, we call num $=\frac{a}{2}\left(\delta_{i}^{2}-\right.$ $\left.\delta_{i-1}^{2}\right)$ and den $=\left(V_{i}-V_{i-1}\right)+a\left(\delta_{i}-\delta_{i-1}\right)$.

- If $d e n=0$ there is no solution. Let us define

$$
\begin{aligned}
& \text { inf }_{3}=\text { sup }_{2}, \\
& \text { sup }_{3}=\sup _{2} .
\end{aligned}
$$

- If den > 0, (57) and (58) hold if and only if

$$
\begin{aligned}
& \text { den } \cdot \max \left\{\delta_{i}, \delta_{i-1}\right\}-\text { num } \leq s_{i} \leq \\
& \leq \text { den } \cdot \min \left\{\frac{V_{i}}{a}+\delta_{i}, T_{i-1}\left(\overline{l_{i-1}}\right)\right\}-\text { num }
\end{aligned}
$$

And so let us define

$$
\begin{aligned}
& \text { inf }_{3}=\text { den } \cdot \max \left\{\delta_{i}, \delta_{i-1}\right\}-\text { num } \\
& \text { sup }_{3}=\text { den } \cdot \min \left\{\frac{V_{i}}{a}+\delta_{i}, T_{i-1}\left(\overline{l_{i-1}}\right)\right\}-\operatorname{num}(65
\end{aligned}
$$


- If den $<0,(57)$ and (58) hold if and only if

$$
\begin{aligned}
& \text { den } \cdot \min \left\{\frac{V_{i}}{a}+\delta_{i}, T_{i-1}\left(\overline{l_{i-1}}\right)\right\}-\text { num } \leq s_{i} \leq \\
& \leq \text { den } \cdot \max \left\{\delta_{i}, \delta_{i-1}\right\}-\text { num. }
\end{aligned}
$$

Then, let us define

$$
\begin{aligned}
& \text { inf }_{3}=\text { den } \cdot \min \left\{\frac{V_{i}}{a}+\delta_{i}, T_{i-1}\left(\overline{l_{i-1}}\right)\right\}-\text { num,(67) } \\
& \text { sup }_{3}=\text { den } \cdot \max \left\{\delta_{i}, \delta_{i-1}\right\}-\text { num. }
\end{aligned}
$$

(4) Collision when vehicle $C_{i-1}$ has stopped $\left(d_{c_{4}, i}\right)$. The preceding vehicle has been able to stop safely but a rear collision still occurs.

In this case $s_{i}$ should directly satisfy $s_{i} \leq d_{s, i}-\overline{l_{i-1}}$. We set

$$
\begin{aligned}
\inf _{4} & =\sup _{3} \\
\sup _{4} & =d_{s, i}-\overline{l_{i-1}} .
\end{aligned}
$$

\section{APPENDIX B. COMPUTATION OF THE DISTANCE TRAVELED BY A VEHICLE FOR VARIABLE VELOCITY AND DECELERATION}

Let us recall that when velocity and deceleration are not constant the collisions may occur in three different ways: (1) vehicles $C_{i}$ and $C_{i-1}$ have not started to brake; (2) both of them are already braking; or (3) vehicle $C_{i-1}$ has stopped, with their respective actual distances to stop $d_{c_{1}, i}, d_{c_{2}, i}$ and $d_{c_{3}, i}$. These distances are computed using the same procedure of Appendix A.

(1) Collision when the vehicles have not started to brake $\left(d_{c_{1}, i}\right)$. This event may happen if the difference of initial velocities makes the vehicles crash before receiving the warning message.

A time instant $t_{c_{1}, i}\left(s_{i}\right)$ should exist so that

$$
\begin{gathered}
V_{i} t_{c_{1}, i}\left(s_{i}\right)=V_{i-1} t_{c_{1}, i}\left(s_{i}\right)+s_{i}, \\
0 \leq t_{c_{1}, i}\left(s_{i}\right) \leq \delta .
\end{gathered}
$$

Solving (71) we have

$$
t_{c_{1}, i}\left(s_{i}\right)=\frac{s_{i}}{V_{i}-V_{i-1}} .
$$

Now, it remains to compute the integration limits $i n f_{1}$ and sup $_{1}$ :

- If $V_{i}-V_{i-1} \leq 0$, there is no solution (no collision can occur). Let us define appropriate limits

$$
\begin{aligned}
& \text { inf }_{1}=0, \\
& \sup _{1}=0 .
\end{aligned}
$$

- If $V_{i}-V_{i-1}>0$, then (72) holds if and only if $0 \leq s_{i} \leq$ $\delta\left(V_{i}-V_{i-1}\right)$. Let us define

$$
\begin{aligned}
& \inf _{1}=0, \\
& \sup _{1}=\delta\left(V_{i}-V_{i-1}\right) .
\end{aligned}
$$

(2) Collision when both vehicles are braking $\left(d_{c_{2}, i}\right)$. A time instant $t_{c_{2}, i}\left(s_{i}\right)$ should exist so that

$$
\begin{aligned}
& V_{i} t_{c_{2}, i}\left(s_{i}\right)-\frac{a_{i}}{2}\left(t_{c_{2}, i}\left(s_{i}\right)-\delta\right)^{2}= \\
& =V_{i-1} t_{c_{2}, i}\left(s_{i}\right)-\frac{a_{i-1}}{2}\left(t_{c_{2}, i}\left(s_{i}\right)-\delta\right)^{2}+s_{i}, \\
& \delta \leq t_{c_{2}, i}\left(s_{i}\right) \leq \min \left\{\frac{V_{i}}{a_{i}}+\delta, T_{i-1}\left(\overline{l_{i-1}}\right)\right\} .
\end{aligned}
$$

In order to simplify the notation, we call $\min =$ $\min \left\{\frac{V_{i}}{a_{i}}+\delta, T_{i-1}\left(\overline{l_{i-1}}\right)\right\}-\delta=\min \left\{\frac{V_{i}}{a_{i}}, T_{i-1}\left(\overline{l_{i-1}}\right)-\delta\right\}$.

- If $a_{i-1}-a_{i}=0$ solving (78), we obtain

$$
t_{c_{2}, i}\left(s_{i}\right)=\frac{s_{i}}{V_{i}-V_{i-1}} .
$$

- If $V_{i}-V_{i-1} \leq 0$, there is no solution. Let us define

$$
\begin{aligned}
& \text { inf }_{2}=\text { sup }_{1}, \\
& \text { sup }_{2}=\text { sup }_{1} .
\end{aligned}
$$

- If $V_{i}-V_{i-1}>0$, (79) holds if and only if

$$
\delta\left(V_{i}-V_{i-1}\right) \leq s_{i} \leq\left(V_{i}-V_{i-1}\right) \cdot \min .
$$

And so let us define

$$
\begin{aligned}
& \text { inf }_{2}=\delta\left(V_{i}-V_{i-1}\right), \\
& \text { sup }_{2}=\left(V_{i}-V_{i-1}\right) \cdot \text { min. }
\end{aligned}
$$

- If $a_{i-1}-a_{i} \neq 0$ solving (78), we obtain the following solutions

$$
\begin{aligned}
t 1_{c_{2}, i}\left(s_{i}\right) & =\frac{V_{i}-V_{i-1}}{a_{i}-a_{i-1}}+\delta- \\
- & \sqrt{\left(\frac{V_{i}-V_{i-1}}{a_{i}-a_{i-1}}+\delta\right)^{2}-\delta^{2}+\frac{2 s(i)}{a_{i-1}-a_{i}}}, \\
t 2_{c_{2}, i}\left(s_{i}\right) & =\frac{V_{i}-V_{i-1}}{a_{i}-a_{i-1}}+\delta+ \\
+ & \sqrt{\left(\frac{V_{i}-V_{i-1}}{a_{i}-a_{i-1}}+\delta\right)^{2}-\delta^{2}+\frac{2 s(i)}{a_{i-1}-a_{i}}} .
\end{aligned}
$$

The term in the square root is positive if and only if $\frac{s_{i}}{\left(a_{i-1}-a_{i}\right)} \geq \frac{\delta\left(V_{i}-V_{i-1}\right)}{\left(a_{i-1}-a_{i}\right)}-\frac{\left(V_{i}-V_{i-1}\right)^{2}}{2\left(a_{i-1}-a_{i}\right)^{2}}$.

First we compute the limits for $t_{1}$ :

If $a_{i-1}-a_{i}>0$, (79) does not hold for $t 1_{c_{2}, i}\left(s_{i}\right)$, and so let us define

$$
\begin{aligned}
& \inf _{2 a}=\sup _{1}, \\
& \sup _{2 a}=\text { sup }_{1} .
\end{aligned}
$$

If $a_{i-1}-a_{i}<0$, then

- If $\frac{V_{i}-V_{i-1}}{a_{i}-a_{i-1}} \leq \min$, then (79) holds for $t 1_{c_{2}, i}\left(s_{i}\right)$ if and only if

$$
\delta\left(V_{i}-V_{i-1}\right) \leq s_{i} \leq \delta\left(V_{i}-V_{i-1}\right)-\frac{\left(V_{i}-V_{i-1}\right)^{2}}{2\left(a_{i-1}-a_{i}\right)} .
$$


Let us define

$$
\begin{aligned}
\inf _{2 a} & =\delta\left(V_{i}-V_{i-1}\right), \\
\sup _{2 a} & =\delta\left(V_{i}-V_{i-1}\right)-\frac{\left(V_{i}-V_{i-1}\right)^{2}}{2\left(a_{i-1}-a_{i}\right)} .
\end{aligned}
$$

- If $\frac{V_{i}-V_{i-1}}{a_{i}-a_{i-1}}>\min$, then (79) holds for $t 1_{c_{2}, i}\left(s_{i}\right)$ if and only if

$$
\begin{aligned}
& \delta\left(V_{i}-V_{i-1}\right) \leq s_{i} \leq \frac{a_{i-1}-a_{i}}{2} \cdot m_{i n}^{2}+ \\
& +\left(V_{i}-V_{i-1}\right) \cdot \min +\delta\left(V_{i}-V_{i-1}\right) .
\end{aligned}
$$

Let us define

$$
\begin{aligned}
\inf _{2 a}= & \delta\left(V_{i}-V_{i-1}\right), \\
\sup _{2 a}= & \min \left\{\delta\left(V_{i}-V_{i-1}\right)-\frac{\left(V_{i}-V_{i-1}\right)^{2}}{2\left(a_{i-1}-a_{i}\right)}\right. \\
& \frac{a_{i-1}-a_{i}}{2} \min ^{2}+\left(V_{i}-V_{i-1}\right) \cdot \min + \\
& \left.+\delta\left(V_{i}-V_{i-1}\right)\right\} .
\end{aligned}
$$

It remains to compute the limits for $t 2_{c_{2}, i}\left(s_{i}\right)$ :

If $\frac{V_{i}-V_{i-1}}{a_{i}-a_{i-1}}>\min$, (79) does not hold for $t 2_{c_{2}, i}\left(s_{i}\right)$, and so let us define

$$
\begin{aligned}
\inf _{2 b} & =\sup _{2 a}, \\
\sup _{2 b} & =\sup _{2 a} .
\end{aligned}
$$

If $\frac{V_{i}-V_{i-1}}{a_{i}-a_{i-1}} \leq \min$, then

- If $a_{i-1}-a_{i}>0$ and $V_{i}-V_{i-1}>0$, then (79) holds for $t 2_{c_{2}, i}\left(s_{i}\right)$ if and only if

$$
\begin{aligned}
& \delta\left(V_{i}-V_{i-1}\right) \leq s_{i} \leq \frac{a_{i-1}-a_{i}}{2} \cdot m_{i n}{ }^{2}+ \\
& +\left(V_{i}-V_{i-1}\right) \cdot \min +\delta\left(V_{i}-V_{i-1}\right) .
\end{aligned}
$$

Let us define

$$
\begin{aligned}
& \inf _{2 b}=\delta\left(V_{i}-V_{i-1}\right), \\
& \sup _{2 b}=\frac{a_{i-1}-a_{i}}{2} \cdot \min ^{2}+ \\
& \quad+\left(V_{i}-V_{i-1}\right) \min +\delta\left(V_{i}-V_{i-1}\right) .
\end{aligned}
$$

- If $a_{i-1}-a_{i}>0$ and $V_{i}-V_{i-1}<0$, then (79) holds for $t 2_{c_{2}, i}\left(s_{i}\right)$ if and only if

$$
\begin{aligned}
& \delta\left(V_{i}-V_{i-1}\right)-\frac{\left(V_{i}-V_{i-1}\right)^{2}}{2\left(a_{i-1}-a_{i}\right)} \leq s_{i} \leq \\
& \leq \frac{a_{i-1}-a_{i}}{2} \cdot \mathrm{min}^{2}+\left(V_{i}-V_{i-1}\right) \cdot \min + \\
& +\delta\left(V_{i}-V_{i-1}\right) .
\end{aligned}
$$

Let us define

$$
\begin{aligned}
& \inf _{2 b}=\delta\left(V_{i}-V_{i-1}\right)-\frac{\left(V_{i}-V_{i-1}\right)^{2}}{2\left(a_{i-1}-a_{i}\right)}, \\
& \sup _{2 b}=\frac{a_{i-1}-a_{i}}{2} \cdot \min ^{2}+ \\
& \quad+\left(V_{i}-V_{i-1}\right) \min +\delta\left(V_{i}-V_{i-1}\right) .
\end{aligned}
$$

- If $a_{i-1}-a_{i}<0$ and $V_{i}-V_{i-1}>0$, then (79) holds for $t 2_{c_{2}, i}\left(s_{i}\right)$ if and only if

$$
\begin{aligned}
& \frac{a_{i-1}-a_{i}}{2} \cdot \min ^{2}+\left(V_{i}-V_{i-1}\right) \cdot \min + \\
& +\delta\left(V_{i}-V_{i-1}\right) \leq s_{i} \leq \delta\left(V_{i}-V_{i-1}\right)- \\
& -\frac{\left(V_{i}-V_{i-1}\right)^{2}}{2\left(a_{i-1}-a_{i}\right)}
\end{aligned}
$$

Let us define

$$
\begin{aligned}
\inf _{2 b} & =\frac{a_{i-1}-a_{i}}{2} \cdot \min ^{2}+ \\
& +\left(V_{i}-V_{i-1}\right) m i n+\delta\left(V_{i}-V_{i-1}\right) \\
\sup _{2 b} & =\delta\left(V_{i}-V_{i-1}\right)-\frac{\left(V_{i}-V_{i-1}\right)^{2}}{2\left(a_{i-1}-a_{i}\right)}
\end{aligned}
$$

- Otherwise, there is no solution, and so let us define

$$
\begin{aligned}
\text { inf }_{2 b} & =\sup _{2 a}, \\
\sup _{2 b} & =\sup _{2 a} .
\end{aligned}
$$

(3) Collision when vehicle $C_{i-1}$ has stopped $\left(d_{c_{3}, i}\right)$. The preceding vehicle has been able to stop safely but a rear-end collision occurs.

In this case $s_{i}$ should directly satisfy that $s_{i} \leq d_{s, i}-\overline{l_{i-1}}$. We set

$$
\begin{aligned}
& \inf _{3}=\sup _{2} \\
& \sup _{3}=d_{s, i}-\overline{l_{i-1}} .
\end{aligned}
$$

\section{REFERENCES}

[1] H. Hartenstein and K.P. Laberteaux, "VANET. Vehicular Applications and Inter-Networking Technologies," Wiley, 2010.

[2] J. Glimm and R.E. Fenton, "An Accident-Severity Analysis for a Uniform-Spacing Headway Policy," IEEE Transactions on Vehicular Technology, vol. 29, no. 1, pp. 96-103, Feb. 1980.

[3] H.S. Tan and J. Huang, "DGPS-Based Vehicle-to-Vehicle Cooperative Collision Warning: Engineering Feasibility Viewpoints," IEEE Transactions on Intelligent Transportation Systems, vol. 7, no. 4, pp. 415-428, Dec. 2006.

[4] C.L. Huang, Y.P. Fallah, R. Sengupta and H. Krishnan, "Intervehicle Transmission Rate Control for Cooperative Active Safety System," IEEE Transactions on Intelligent Transportation Systems, vol. 12, no. 3, pp. 645-658, Sept. 2011.

[5] A. Vahidi and A. Eskandarian, "Research advances in intelligent collision avoidance and adaptive cruise control," IEEE Transactions on Intelligent Transportation Systems, vol. 4, no. 3, pp. 143-153, Sept. 2003.

[6] T. Kim and H.Y. Jeong, "Crash Probability and Error Rates for HeadOn Collisions Based on Stochastic Analyses," IEEE Transactions on Intelligent Transportation Systems, vol. 11, no. 4, pp. 896-904, Dec. 2010.

[7] G. Grime and I.S. Jones, "Car collisions-the movement of cars and their occupants in accidents," Proceedings of the Institute of Mechanical Engineers, vol. 184, no. 5, pp. 87-136, 1969.

[8] A. Touran, M.A. Brackstone and M. McDonald, "A collision model for safety evaluation of autonomous intelligent cruise control," Accident Analysis and Prevention, vol. 31, no. 5, pp. 567-578, Sept. 1999.

[9] M. Treiber, A. Hennecke and D. Helbing, "Congested traffic states in empirical observations and microscopic simulations," Physical Review E, vol. 62, no. 2, pp. 1805-1824, Aug. 2000.

[10] A. Chakravarthy, K. Song and E. Feron, "Preventing Automotive Pileup Crashes in Mixed-Communication Environments," IEEE Transactions on Intelligent Transportation Systems, vol. 10, no. 2, pp. 211-225, June 2009. 
[11] Y. Yang and T.P. Yum, "Delay Distributions of Slotted ALOHA and CSMA," IEEE Transactions on Communications, vol. 51, no. 11, pp. 1846-1857, Nov. 2003.

[12] M.A. Abdel-Aty and A.E. Radwan, "Modeling traffic accident occurrence and involvement," Accident Analysis and Prevention, vol. 32, no. 5, pp. 633-642, Sept. 2000

[13] J.J. Song, M. Ghosh, S. Miaou and B. Mallick, "Bayesian multivariate spatial models for roadway traffic crash mapping," Journal of Multivariate Analysis, vol. 97, no. 1, pp. 246-273, Jan. 2006.

[14] N. Wisitpongphan, F. Bai, P. Mudalige, V. Sadekar and O. Tonguz, "Routing in Sparse Vehicular Ad Hoc Wireless Networks," IEEE Journal on Selected Areas in Communications, vol. 25, no. 8, pp. 1538-1556, Oct. 2007.

[15] T. Taleb, A. Benslimane and K. Ben Letaief, "Toward an Effective RiskConscious and Collaborative Vehicular Collision Avoidance System," IEEE Transactions on Vehicular Technology, vol. 59, no. 3, pp. 14741486, March 2010.

[16] S. Biswas, R. Tatchikou and F. Dion, "Vehicle-to-Vehicle Wireless Communication Protocols for Enhancing Highway Traffic Safety," IEEE Communications Magazine, vol. 44, no. 1, pp. 74-82, Jan. 2006.

[17] "IEEE Standard for Wireless Access in Vehicular Environments (WAVE) - Multi-Channel Operation," IEEE Std. 1609.4-2010, Feb. 2011.

[18] G. Johansson and K. Rumar, "Driver's Brake Reaction Times," Human Factors and Ergonomics Society, vol. 13, no. 1, pp. 23-27, Feb. 1971.

[19] E. Bertolazzi, F., Biral, M. Da Lio, A. Saroldi and F. Tango, "Supporting Drivers in Keeping Safe Speed and Safe Distance: The SASPENCE Subproject Within the European Framework Programme 6 Integrating Project PReVENT", IEEE Transactions on Intelligent Transportation Systems, vol. 11, no. 3, pp. 525-538, Sept. 2010.

[20] J. Lee, D. McGehee, T. Brown and M. Reyes, "Collision Warning Timing, Driver Distraction, and Driver Response to Imminent Rear-End Collisions in a High-Fidelity Driving Simulator," Human Factors: The Journal of the Human Factors and Ergonomics Society, vol. 44 no. 2, pp. 314-334, 2002.

[21] K. Yi and J. Chung, "Nonlinear brake control for vehicle CW/CA systems," IEEE/ASME Transactions on Mechatronics, vol. 6, no. 1, pp. 17-25, Mar 2001.

[22] T. Wada, S. Doi, N. Tsuru, K. Isaji and H. Kaneko, "Characterization of Expert Drivers' Last-Second Braking and Its Application to a Collision Avoidance System", IEEE Transactions on Intelligent Transportation Systems, vol. 11, no. 2, pp. 413-422, June 2010

[23] M. Torrent-Moreno, J. Mittag, P. Santi and H. Hartenstein, "Vehicle-tovehicle Communication: Fair Transmit Power Control for Safety-Critical Information," IEEE Transactions on Vehicular Technology, vol. 58, no. 7, pp. 3684-3703, Sept. 2009

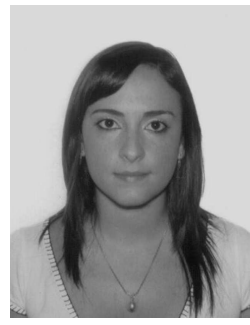

Carolina Garcia-Costa received the Mathematics degree in 2008 and the Master degree in Advanced Mathematics in 2009 from the University of Murcia, Spain. She is currently working toward a Ph.D. degree at the Department of Information Technologies and Communications, Universidad Politécnica de Cartagena (UPCT). Her current research interests are focused on Vehicular Ad-hoc Networks, particularly on crash-probability estimation, active safety systems and performance analysis of medium access control (MAC) protocols

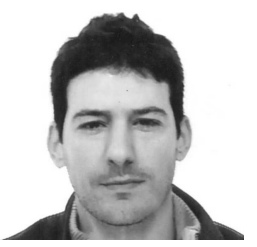

Esteban Egea-Lopez received the Telecommunications Engineering degree in 2000, from Universidad Politécnica de Valencia (UPV), Spain, the Master Degree in Electronics in 2001, from the University of Gavle, Sweden, and Ph.D. in Telecommunications in 2006 from Universidad Politécnica de Cartagena (UPCT). Since 2001, he is an assistant professor of the Department of Information Technologies and Communications at UPCT. His research interest is focused on RFID, Vehicular, Ad-hoc and wireless sensor networks.

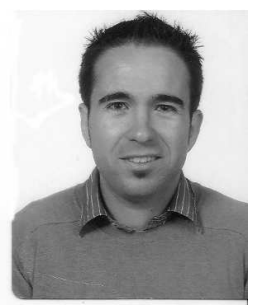

Juan Bautista Tomas-Gabarron received the B.S. Degree in Telecommunications Engineering from Universidad Politécnica de Cartagena (UPCT) in 2008, Murcia, Spain (Final Work Project developed in conjunction with the Technical University of Munich, Germany). He received the Master of Research in Communications in October 2009 at Universida Politécnica de Cartagena (UPCT). Currently, he is $\mathrm{Ph} . \mathrm{D}$ Student at UPCT. His research interests are Vehicular Ad-hoc Networks, in particular the design, evaluation and testing of CCA (Cooperative Collision Avoidance) applications to develop communication protocols which can minimize the probability of accident in critical emergency brake situations.

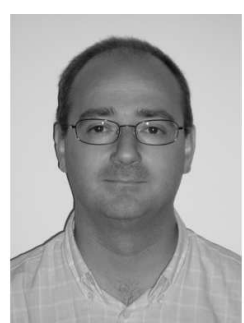

(1995).
Joan Garcia-Haro is a Professor at Universidad Politécnica de Cartagena (UPCT), Spain. He is author or co-author of more than 60 journal papers mainly in the fields of switching, wireless networking and performance evaluation. From April 2002 to December 2004 he served as EIC of the IEEE Global Communications Newsletter, included in the IEEE Communications Magazine. He is Technical Editor of the same magazine from March 2001. He also holds an Honorable Mention for the IEEE Communications Society Best Tutorial paper Award

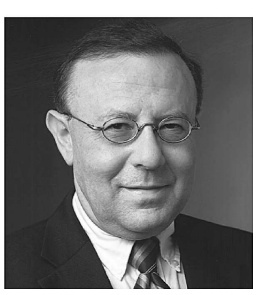

Zygmunt J. Haas received his B.Sc. in EE in 1979 and M.Sc. in EE in 1985. In 1988, he earned his $\mathrm{Ph} . \mathrm{D}$. from Stanford University and subsequently joined AT\&T Bell Laboratories in the Network Research Department. There he pursued research on wireless communications, mobility management, fast protocols, optical networks, and optical switching. From September 1994 till July 1995, Dr. Haas worked for the AT\&T Wireless Center of Excellence, where he investigated various aspects of wireless and mobile networking, concentrating on TCP/IP networks. As of August 1995, he joined the faculty of the School of Electrical and Computer Engineering at Cornell University, where he is now a Professor.

Dr. Haas is an author of numerous technical papers and holds eighteen patents in the fields of high-speed networking, wireless networks, and optical switching. He has organized several workshops, delivered numerous tutorials at major IEEE and ACM conferences, and has served as editor of several journals and magazines, including the IEEE Transactions on Networking, the IEEE Transactions on Wireless Communications, the IEEE Communications Magazine, the Springer Wireless Networks journal, the Elsevier Ad Hoc Networks journal, the Journal of High Speed Networks, and the Wiley Wireless Communications and Mobile Computing journal. He has been a guest editor of IEEE JSAC issues on "Gigabit Networks," "Mobile Computing Networks," and "Ad-Hoc Networks." Dr. Haas is an IEEE Fellow and a voting member of ACM. He has served in the past as a Chair of the IEEE Technical Committee on Personal Communications (TCPC). His interests include: mobile and wireless communication and networks, biologicallyinspired networks, and modeling of complex systems. 